\title{
Review Article \\ Beyond Standard Model Searches in the MiniBooNE Experiment
}

\author{
Teppei Katori ${ }^{1}$ and Janet M. Conrad ${ }^{2}$ \\ ${ }^{1}$ Queen Mary University of London, London E1 4NS, UK \\ ${ }^{2}$ Massachusetts Institute of Technology, Cambridge, MA 02139, USA \\ Correspondence should be addressed to Teppei Katori; t.katori@qmul.ac.uk
}

Received 31 March 2014; Accepted 5 August 2014

Academic Editor: Abhijit Samanta

Copyright (C) 2015 T. Katori and J. M. Conrad. This is an open access article distributed under the Creative Commons Attribution License, which permits unrestricted use, distribution, and reproduction in any medium, provided the original work is properly cited. The publication of this article was funded by SCOAP ${ }^{3}$.

\begin{abstract}
The MiniBooNE experiment has contributed substantially to beyond standard model searches in the neutrino sector. The experiment was originally designed to test the $\Delta m^{2} \sim 1 \mathrm{eV}^{2}$ region of the sterile neutrino hypothesis by observing $\nu_{e}\left(\bar{\nu}_{e}\right)$ charged current quasielastic signals from a $\nu_{\mu}\left(\bar{\nu}_{\mu}\right)$ beam. MiniBooNE observed excesses of $v_{e}$ and $\bar{\nu}_{e}$ candidate events in neutrino and antineutrino mode, respectively. To date, these excesses have not been explained within the neutrino standard model ( $\nu \mathrm{SM})$; the standard model extended for three massive neutrinos. Confirmation is required by future experiments such as MicroBooNE. MiniBooNE also provided an opportunity for precision studies of Lorentz violation. The results set strict limits for the first time on several parameters of the standard-model extension, the generic formalism for considering Lorentz violation. Most recently, an extension to MiniBooNE running, with a beam tuned in beam-dump mode, is being performed to search for dark sector particles. This review describes these studies, demonstrating that short baseline neutrino experiments are rich environments in new physics searches.
\end{abstract}

\section{Introduction}

Across the particle physics community, the mysterious periodic-table-like nature of the standard model (SM) is motivating searches for new particles, new forces, and new properties of the particles that are known. The neutrino sector is proving a rich environment for these searches. Having already found one beyond standard model (BSM) effect, neutrino mass [1], a series of experiments are pursuing other potential signals. Unlike the case of three-neutrino oscillation measurements within $\nu \mathrm{SM}$, many of these searches are pursued over short baselines, from a few meters to approximately a kilometer. The Mini Booster Neutrino Experiment (MiniBooNE) at Fermi National Accelerator Laboratory (Fermilab) is an excellent example, having contributed substantially to BSM studies.

This review describes the MiniBooNE BSM program. We begin by describing the experiment. This is followed by a discussion of the MiniBooNE cross section studies, which have been essential input to both the BSM searches within this experiment and also to other experiments, including T2K most recently [2]. We then describe three searches: the sterile neutrino search which motivated the experiment, Lorentz violation searches which set the first limits on five neutrino sector parameters, and the search for dark sector particles which is now being pursued with a reconfigured beam.

\section{MiniBooNE Experiment}

MiniBooNE (running from 2002 to 2012) was originally designed to test the LSND signal [3]. In the LSND experiment, low energy ( 0 to $53 \mathrm{MeV}$ ) muon antineutrinos were produced by pion decay-at-rest (DAR) and were detected by the liquid-scintillator-based LSND detector at $31 \mathrm{~m}$ from the target. The observed $3.8 \sigma$ excess of $\bar{\nu}_{e}$ candidate events could be interpreted as oscillations in the $\Delta m^{2} \sim 1 \mathrm{eV}^{2}$ region within a simple two massive neutrino oscillation hypothesis, where the oscillation probability is given by

$$
P\left(\bar{v}_{\mu} \longrightarrow \bar{\nu}_{e}\right)=\sin ^{2} 2 \theta \sin ^{2}\left(\frac{1.27 \Delta m^{2} L}{E}\right) .
$$




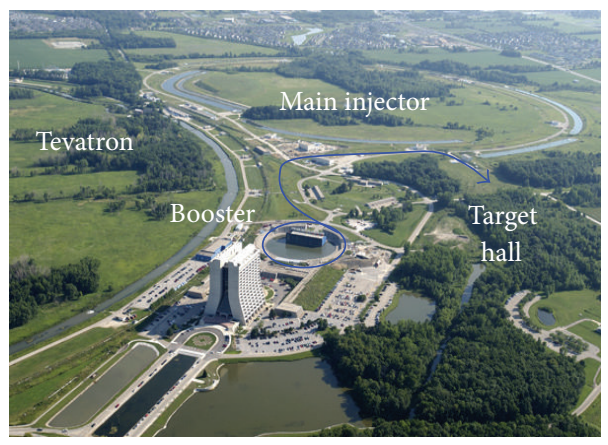

(a)

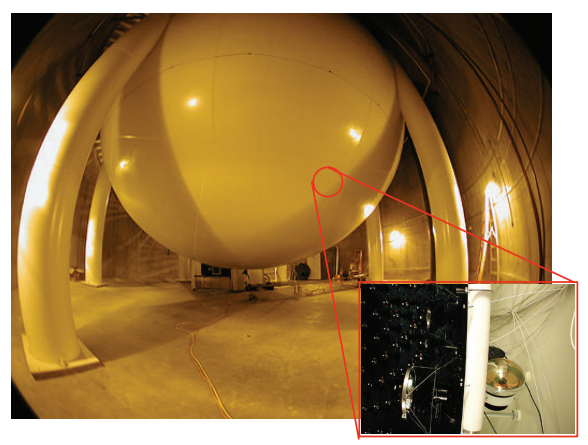

(b)

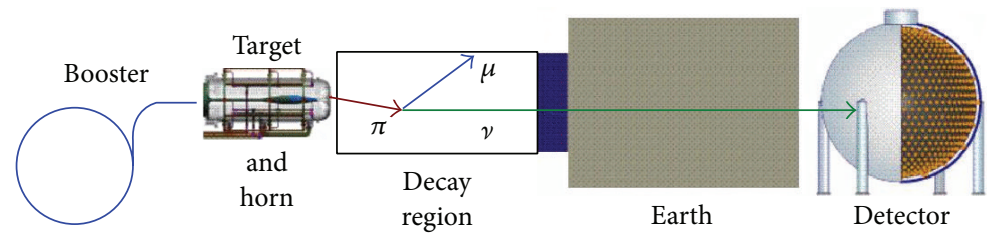

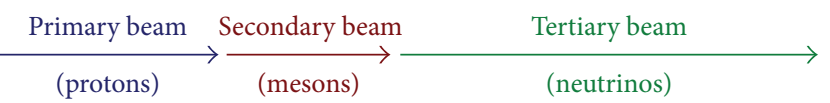

(c)

Figure 1: The MiniBooNE experiment layout [4]. (a) The Fermilab accelerator complex. (b) The MiniBooNE detector, with inset showing the black inner volume and the white outer volume. (c) Schematic layout of the beam and detector [18].

Here, $\theta$ and $\Delta m^{2}$ are oscillation parameters to control the amplitude and the period, respectively (further discussed in Section 4$), L$ is the distance from neutrino production to interaction in meters, and $E$ is the energy of the neutrino in $\mathrm{MeV}$.

An experiment which maintains the same $L / E$ ratio should observe an oscillation probability consistent with LSND if the simple two neutrino model is a good approximation of the underlying effect. However, by employing an average $E$ which is an order of magnitude larger than LSND, the systematic errors associated with production and decay are quite different. If $L$ is increased accordingly, and no signal is observed, this rules out the two-neutrino oscillation hypothesis of the LSND result.

MiniBooNE was designed with this in mind. The MiniBooNE beam peaked at $\sim 700 \mathrm{MeV}$ and the Cherenkov detector was located at $\sim 500 \mathrm{~m}$ baseline. Figure 1 shows an overview of the MiniBooNE design [4], and in the remainder of this section we provide more details.

\subsection{Booster Neutrino Beam-Line. The Booster Neutrino} Beam-line (BNB) extracts $8 \mathrm{GeV}$ kinetic energy protons from the Fermilab Booster, a $149 \mathrm{~m}$ diameter synchrotron (Figure 1(a)). Eighty-one bunches, separated in time by $\sim 19 \mathrm{~ns}$, are extracted by a fast kicker within a $\sim 1.6 \mu$ s pulse. Each pulse contains around $4 \times 10^{12}$ protons. Typically, four to five pulses per second were sent to $\mathrm{BNB}$ to produce the neutrino beam.

This high intensity proton pulse collides with a beryllium target to produce a shower of mesons (Figure 1(c)). The target is located within a magnetic focusing horn. For neutrino mode running, the toroidal field generated by the horn focuses on positive mesons, with $\pi^{+}$decay-in-flight (DIF) as the primary source of the $\nu_{\mu}$ beam. In antineutrino mode running, the horn focuses on negative mesons to create the $\bar{\nu}_{\mu}$ dominant beam. The details of the BNB neutrino flux prediction can be found in [5].

MiniBooNE collected $6.46 \times 10^{20}$ proton-on-target (POT) in neutrino mode and $11.27 \times 10^{20}$ POT in antineutrino mode.

2.2. The MiniBooNE Detector. The MiniBooNE detector, located $541 \mathrm{~m}$ away from the target, is a mineral-oil-based Cherenkov detector. The $12.2 \mathrm{~m}$ spherical tank, filled with pure mineral oil, $\left(\mathrm{CH}_{2}\right)_{n}$, has two optically separated regions. The interior region, lined by 12808 -inch photomultiplier tubes (PMTs), contains the target volume. An outer volume, equipped with 240 8-inch PMTs, serves as the veto region [6]. The presence of a charged particle above threshold is detected through the Cherenkov radiation observed by PMTs. As seen from Figure 1(b), the inner volume is painted black to prevent scattering of the Cherenkov light, improving the reconstruction precision. On the other hand, the outer volume is painted white to enhance scattering of Cherenkov light, in order to achieve the $99.9 \%$ rejection of cosmic rays by the veto [7] even with fairly sparse PMT coverage. The charge and time information from all PMTs is used to reconstruct kinematics of charged-lepton and electromagnetic events. MiniBooNE mineral oil produces a small amount of scintillation light which can be used to reconstruct the total energy of the interaction via calorimetry, which is particularly important for particles below Cherenkov threshold. 


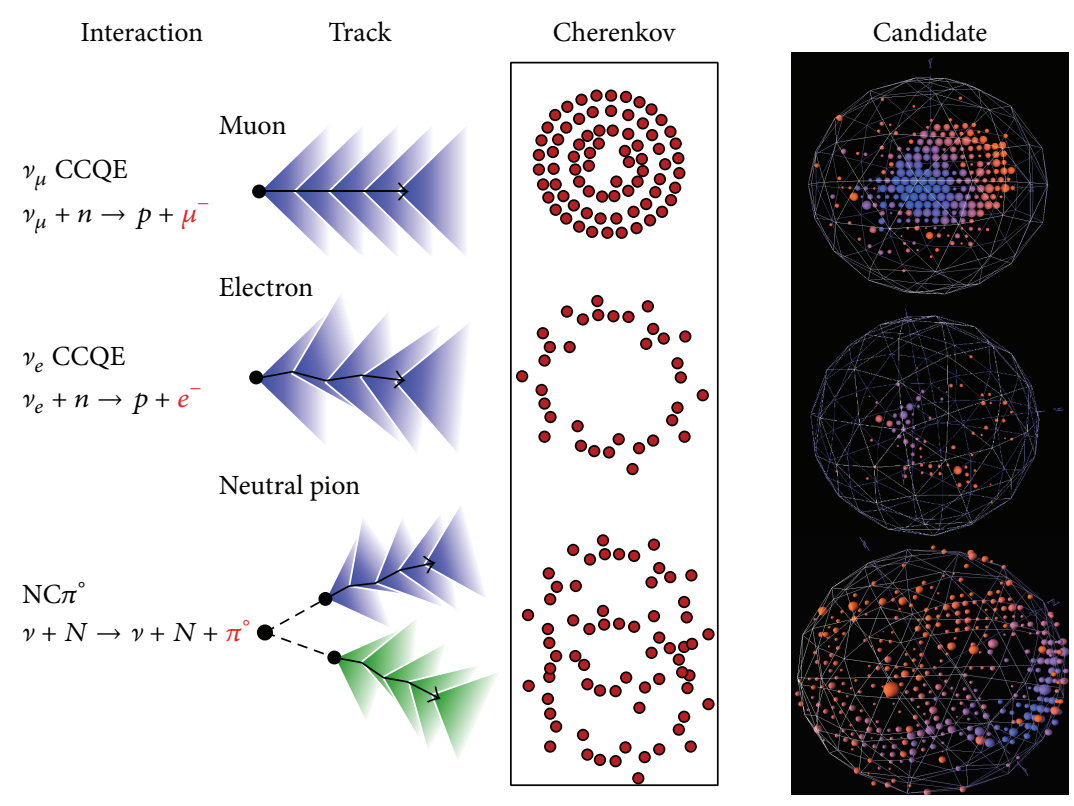

FIgure 2: (Color online) MiniBooNE particle reconstruction [4]. From top to bottom, a muon neutrino charged-current quasielastic (CCQE) interaction, an electron neutrino CCQE interaction, and a neutral current, neutral pion production ( $\left.\mathrm{NCl} \pi^{\circ}\right)$ interaction. The second and the third columns show the characteristics of tracks and Cherenkov rings [7], and the last column shows the event displays of candidate events.

For the $\nu_{\mu} \rightarrow \nu_{e}\left(\bar{\nu}_{\mu} \rightarrow \bar{\nu}_{e}\right)$ oscillation study, the following three particle reconstruction algorithms were the most important: single Cherenkov rings from (1) a muon and (2) an electron and the two-ring electromagnetic shower topology from (3) a neutral pion decay to two gammas. Figure 2 shows the different characteristics of these three signals, including examples of typical events in the detector [4].

The reconstruction algorithms can also reconstruct more complicated topologies important for constraining backgrounds and for cross section studies discussed below. The charged-current single charged pion $\left(\mathrm{CCl} \pi^{+}\right)$interaction reconstruction algorithm [8] fit two Cherenkov rings from final state particles, a charged lepton, and a positive pion, to find their kinematics. The charged-current single neutral pion $\left(\mathrm{CCl} \pi^{\circ}\right)$ interaction reconstruction algorithm [9] fit a charged lepton and a neutral pion (which consists of two electromagnetic showers, that is, the algorithm fits for three Cherenkov rings). Another algorithm identifies and reconstructs the neutral current elastic (NCE) interaction [10], where the total kinetic energy of final state nucleons is found using scintillation light.

Along with reconstruction of the light topology in the detector, event identification also relies upon "subevents." These are bursts of light separated in time which indicate a sequence of decay. For example, a muon which stops and then emits a decay ("Michel") electron will produce two subevents, one from the initial muon and the one from the Michel electron.

\section{MiniBooNE Cross Section Results}

All searches for BSM physics rely on a precise understanding of SM interactions. However, when MiniBooNE began running, there was little neutrino cross section data in the $100 \mathrm{MeV}$ to few $\mathrm{GeV}$ energy regime. In response, MiniBooNE developed a highly successful campaign of cross section measurements, some of which are described here. These results are interesting by themselves and also can be used as direct inputs to the BSM analyses, as described later in this paper.

MiniBooNE's beam is among the first high-statistics, high purity fluxes in the energy range from 100 to $1500 \mathrm{MeV}$. The observation of the resulting events in a large, isotropic detector with $4 \pi$ coverage is unique. Within this detector it is relatively easy to achieve uniform angular acceptance. Also, the active veto makes it possible to measure NC interactions effectively. Insensitivity of hadronic details worked in positively. The hadron multiplicity often causes confusions for tracker detectors. Although the MiniBooNE detector cannot measure multiple hadron tracks, it measures total energy of low energy hadrons (such as protons below Cherenkov threshold from CCQE interactions) in calorimetric way, and, as a result, the details of final state interactions (FSIs), such as rescattering, absorption, and charge exchange, do not strongly affect reconstruction of kinematics.

Perhaps most importantly to the overall impact of the data, the MiniBooNE collaboration provided the cross section data in a form that is most useful to theorists. Traditionally, cross section data have been presented either as a function of neutrino energy $\left(E_{v}\right)$ or 4-momentum transfer $\left(Q^{2}\right)$. This presentation is problematic in the MiniBooNE energy region, because of the importance of nuclear effects: Fermi motion smears the kinematics, binding energy shifts the energy spectrum, nucleon correlations affect both energy dependence and normalization of cross sections, and pions may be created, absorbed, and charge-exchanged within 
the nuclear environment. These nuclear processes modify the features of primary neutrino-nucleon interactions, and so model dependent corrections are required to reconstruct $E_{v}$ and $Q^{2}$. This model dependence is problematic because there are a wide range of models available [11-15].

Instead, MiniBooNE chose to publish flux-integrated differential cross sections in terms of measured kinematic variables, which are essentially model-independent. These results have the detector efficiency unfolded but are presented without any other corrections. In particular, the neutrino flux is not unfolded. The result is data that is neutrino beam specific, and theoretical models are comparable only if those models are convoluted with the MiniBooNE predicted neutrino flux. However, this is trivial for all theorists to do, given that MiniBooNE published a first-principles flux prediction [16]. This isolates all model dependence in the data-to-prediction comparison entirely to the "prediction" side of the discussion. The data remains completely general. For this reason, the MiniBooNE cross section data are widely used to study and compare theoretical models. In this section, we describe each cross section measurement briefly.

3.1. Charged-Current Quasielastic (CCQE) Scattering. The CCQE interaction is the primary interaction at MiniBooNE energies. This interaction is used to detect $\nu_{\mu}\left(\bar{\nu}_{\mu}\right)$ and $\nu_{e}\left(\bar{\nu}_{e}\right)$ candidate events in the oscillation and Lorentz violation analyses:

$$
\begin{aligned}
& \nu_{\mu}+n \longrightarrow \mu^{-}+p, \\
& \bar{\nu}_{\mu}+p \longrightarrow \mu^{+}+n, \\
& \nu_{e}+n \longrightarrow e^{-}+p, \\
& \bar{\nu}_{e}+p \longrightarrow e^{+}+n .
\end{aligned}
$$

Therefore, a strong understanding of this channel is essential. High statistics $\nu_{\mu}\left(\bar{\nu}_{\mu}\right)$ interactions are used to study outgoing lepton kinematics [17]. The observable of this channel is the outgoing muon, with no pions in the final state; that is, the signal event topology is " 1 muon +0 pion $+N$ protons." The main results were published in terms of flux-integrated double differential cross sections, as functions of the lepton kinetic energy and the scattering angle. Figure 3(a) shows the flux-integrated double differential cross section of $\nu_{\mu}$ CCQE interactions [18]. The irreducible background from the pion production channel is subtracted based on a sideband study, but the subtracted background is also published so that readers can recover the irreducible background.

These data have revealed the importance of nucleon correlations $[19,20]$ in neutrino scattering, which had not been taken into account correctly in previous calculations. This led to models developed using electron scattering data that were tested against MiniBooNE data [21-26]. These models await being tested further by other experiments, such as MINERvA [27, 28] and T2K [29].

Another important test is CCQE antineutrino scattering, where a wide range of expectations were predicted prior to the run [30-34]. Before the data could be compared to the results, however, the substantial contamination of neutrinos in the antineutrino beam had to be addressed. Three independent methods were used to constrain and tune the neutrino contamination prediction [35]. After subtracting the neutrino contamination, the flux-integrated double differential cross section for the muon antineutrino CCQE interaction was measured (Figure 3(b)) [36]. The comparison of models with data showed a preference for the high cross section models [37]. The rich shape information of the double differential data continues to provide additional tests, beyond the normalization.

The main result of the $\bar{\nu}_{\mu}$ CCQE cross section measurements is quoted as per $\mathrm{CH}_{2}$ molecule. This is because the MiniBooNE target consists of $\mathrm{CH}_{2}$, and the experiment cannot distinguish antineutrino interactions with bound protons in the carbon nuclei and free protons from hydrogen. As a separate study, however, MiniBooNE also presented an analysis that subtracted the hydrogen interactions, where the cross sections were then expressed per bound proton. This has also provided a useful handle for theorists.

3.2. Charged Single Pion Production. The understanding of charged-current single-pion channels is of great interest to the nuclear community, but also, there are significant implications for the neutrino oscillation studies. These interactions produce an irreducible background for CCQE events [38-41]. If the detector fails to tag outgoing pions, either because of detector effects or nuclear effects, pion production channels may be misclassified as CCQE. The distributions of irreducible backgrounds must be modelled, and those models rely on the pion production measurements, especially the MiniBooNE data described here. Therefore, understanding the kinematic distributions of pion production channels is a crucial task for neutrino oscillation physics.

There are three pion production channels for which MiniBooNE performed dedicated measurements: chargedcurrent single $\pi^{+}\left(\mathrm{CCl} \pi^{+}\right)$production [8]; charged-current single $\pi^{\circ}\left(\mathrm{CC} 1 \pi^{\circ}\right)$ production [9]; and neutral current single $\pi^{\circ}\left(\mathrm{NCl} \pi^{\circ}\right)$ production [42]:

$$
\begin{gathered}
v_{\mu}+\mathrm{CH}_{2} \longrightarrow \mu^{-}+\pi^{+}+X, \\
v_{\mu}+\mathrm{CH}_{2} \longrightarrow \mu^{-}+\pi^{\circ}+X, \\
v_{\mu}\left(\bar{v}_{\mu}\right)+\mathrm{CH}_{2}+\longrightarrow v_{\mu}\left(\bar{v}_{\mu}\right)+\pi^{\circ}+X .
\end{gathered}
$$

Here, the topologies of each event are more complicated and are described as " 1 muon +1 positive pion $+N$ protons" $\left(\mathrm{CCl} \pi^{+}\right)$, "1 muon +1 neutral pion $+N$ protons" $\left(\mathrm{CCl} \pi^{\circ}\right)$, and " 0 muon +1 neutral pion $+N$ protons" $\left(\mathrm{NCl} \pi^{\circ}\right)$. Although the MiniBooNE detector is not magnetized and therefore cannot distinguish positive and negative pions based on their trajectories, separation is possible. Negative pions are absorbed by a nucleus almost $100 \%$ of the time, and in consequence, there is no emission of a Michel electron. This fact allows MiniBooNE to use the presence of a Michel electron to select positive pions. 


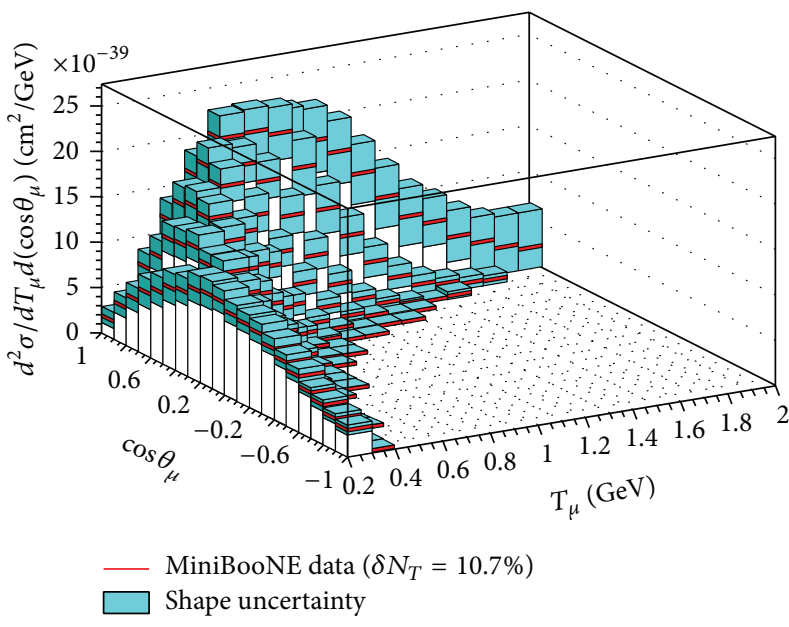

(a)

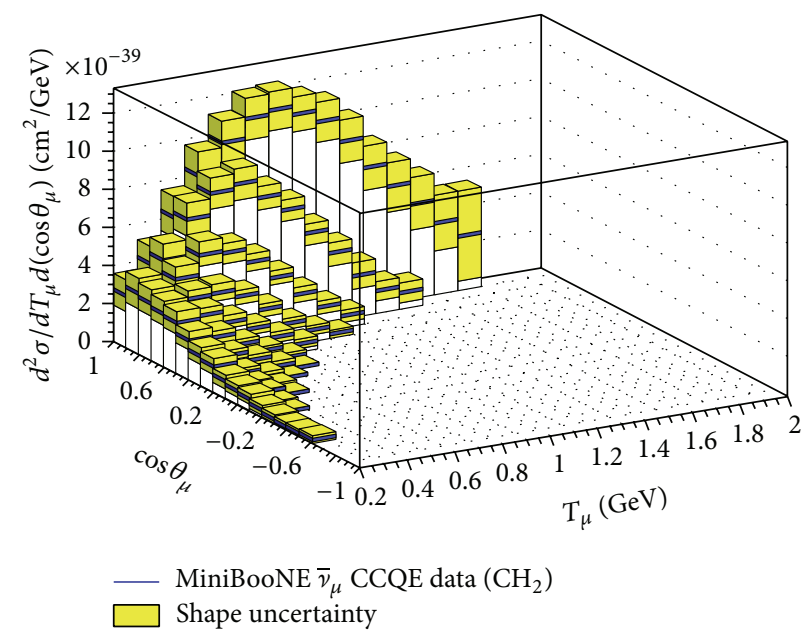

(b)

FIgure 3: (Color online) MiniBooNE CCQE cross sections. (a) shows the muon neutrino flux-integrated CCQE double differential cross section on a neutron target. (b) shows muon antineutrino flux integrated CCQE double differential cross section on a $\mathrm{CH}_{2} \mathrm{molecule}$.

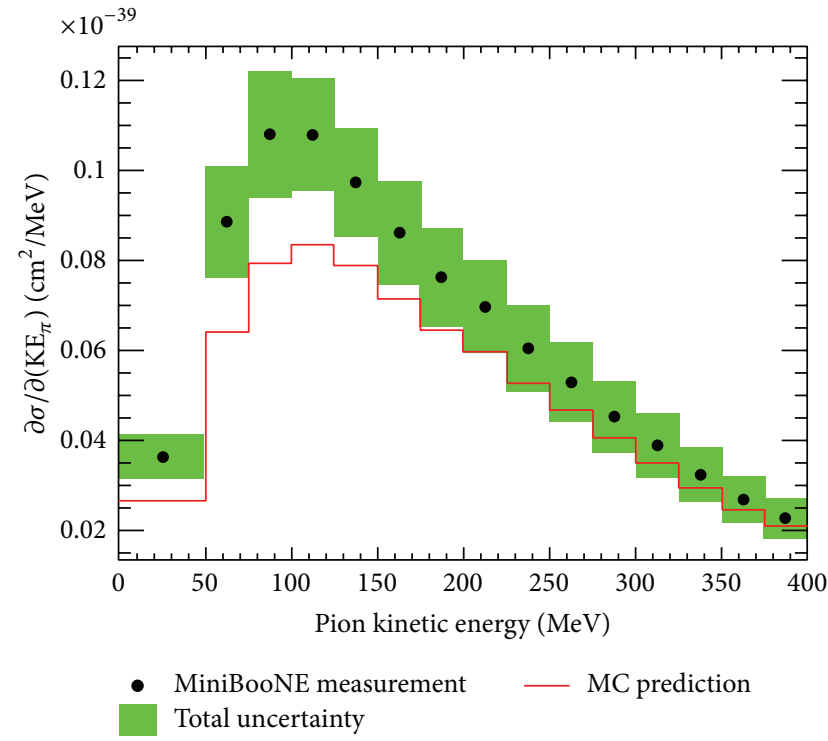

(a)

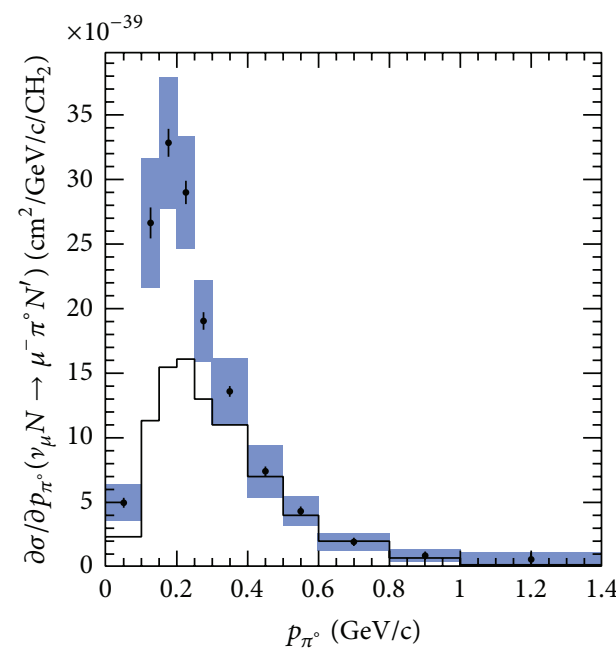

- Statistical error — MC prediction Systematic error

(b)

FIGURE 4: (Color online) MiniBooNE single pion production results. (a) is $\pi^{+}$kinetic energy differential cross section from CC1 $\pi^{+}$interaction on $\mathrm{CH}_{2}$ [8]. (b) is $\pi^{\circ}$ momentum differential cross section from $\mathrm{CCl} \pi^{\circ}$ interaction in $\mathrm{CH}_{2}$ [9]. As you see, predictions underestimate data for both channels and the shapes do not agree as well.

Because of the more complicated topologies, the differential cross sections for these data sets are presented in various variables. Among them, distributions in pion kinetic energy and momentum distributions exhibit the presence of nuclear effects, while we do not see this from the lepton distributions. Figure 4 shows differential cross sections, $\mathrm{CCl} \pi^{+}$pion kinetic energy, and $\mathrm{CCl} \pi^{\circ}$ pion momentum, respectively. The shape and normalization are sensitive to nuclear effects, such as pion absorption, charge exchange, and rescattering. Therefore, the state-of-the-art nuclear models $[43,44]$ can be tested by these MiniBooNE data.
3.3. Neutral Current Elastic (NCE) Scattering. The NCE interaction can take place on both neutrons and protons, for both neutrino and antineutrinos. The results are relevant for dark matter searches in two ways: first through the measurement of $\Delta s$ that we describe here, second as a background to a direct dark matter search by MiniBooNE, described in Section 6:

$$
\begin{aligned}
& \nu_{\mu}\left(\bar{\nu}_{\mu}\right)+p \longrightarrow v_{\mu}\left(\bar{\nu}_{\mu}\right)+p, \\
& \nu_{\mu}\left(\bar{\nu}_{\mu}\right)+n \longrightarrow v_{\mu}\left(\bar{\nu}_{\mu}\right)+n .
\end{aligned}
$$






(a)

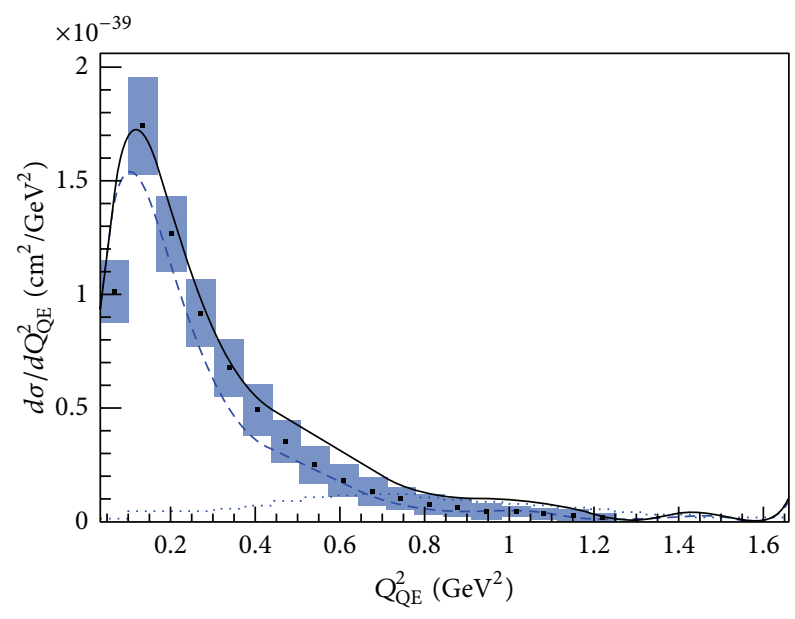

-. MiniBooNE $\bar{v}$ NCE cross section with total error
… MC NCE-like background
- $\operatorname{MC~}\left(M_{A}=1.35 \mathrm{GeV}\right)$
-- $\operatorname{MC~}\left(M_{A}=1.02 \mathrm{GeV}\right)$

(b)

Figure 5: (Color online) MiniBooNE NCE results [45]. (a) shows simulated kinetic energy of protons and neutrons from NCE in MiniBooNE. The line denotes the Cherenkov threshold, that is, only protons which have higher energy from this line emit Cherenkov radiation. (b) shows the antineutrino NCE differential cross section. As you see, the data shows a "roll-over" in the low $Q^{2}$ region.

Since only protons with kinetic energy above $\sim 350 \mathrm{MeV}$ produce Cherenkov radiation (Figure 5(a)), the majority of these events only produce scintillation light and therefore necessitate a strictly calorimetric analysis. For neutrons, there is no Cherenkov radiation and the chance the secondary proton from the primary neutron exceeds this threshold is extremely low (in other words, if the proton exceeds Cherenkov threshold, this will most likely form the primary neutrino NC interaction). We call this topology " 0 muon + 0 pion $+N$ protons." However, when the kinetic energy exceeded the Cherenkov threshold, it is also possible to observe the direction of nucleons [10].

The calorimetric measurement causes the signal to be insensitive to the detailed final state interaction (FSI) process. Also, similar to the antineutrino CCQE analysis (Section 3.1), scattering on $\mathrm{C}$ and $\mathrm{H}$ cannot be distinguished, so the target may be a bound proton, a free proton, or a bound neutron. Hence, the cross section is presented per $\mathrm{CH}_{2}$ target. Figure 5(b) shows the antineutrino mode NCE differential cross section [45].

The NCE data allows us to refine our understanding of nuclear effects at low $Q^{2}$. In NCE, the observable is the sum of all kinetic energies of outgoing protons, $\sum T_{N}$. Using this, the $Q^{2}$ can be reconstructed by assuming the target nucleon at rest:

$$
Q_{\mathrm{QE}}^{2}=2 M_{N} \sum T_{N}
$$

Note that irreducible backgrounds, such as NC pion production without an outgoing pion, are subtracted to make $Q_{\mathrm{QE}}^{2}$ physical.
The reconstructed data shows a roll-over at the low $Q^{2}$ region, due to the combination of Pauli blocking and the nuclear shadowing. Pauli blocking is a phenomenon where low momentum transfer interactions are forbidden due to occupied phase space, and the nuclear shadowing happens when the resolution (= low momentum transfer interaction) is insufficient to resolve a single nucleon wave function. Note that these nuclear effects do not appear if the signal of NCE is defined to be a single isolated proton, where strong FSI migrates all nucleons to low energy region [46]. However, because the MiniBooNE NCE data presents the sum of the total nucleon kinetic energy, the results preserve the feature of the primary neutrino interaction physics.

NCE interactions are connected to direct dark matter searches through the measurement of $\Delta s$, the spin of the strange quarks in the nucleon. It has been shown [47] that the uncertainty of $\Delta s$ on the spin-dependent scattering between dark matter particles and target nuclei can be a large systematic error. Therefore, a $\Delta s$ measurement is another way that neutrino cross section measurements contribute to BSM physics. We briefly consider how this information can be extracted from the NCE data here.

The spin structure of a nucleon is deeply fundamental and quite complicated. In the naive constituent quark model, the spin $-1 / 2$ of a nucleon can be derived by adding valence quark spins, where in the static limit $\left(Q^{2} \rightarrow 0\right)$ there are three valence quarks that make up all static properties of a nucleon, such as charge, magnetic moment, and spin. However, the spin contribution from up and down quarks deduced from inclusive deep inelastic scattering (DIS) measurements [4850] indicates, in the static limit, that up and down quarks support only $\sim 10 \%$ of the total spin of a proton. This so-called 
"spin crisis" has triggered a world wide effort to look for other sources of spin in a nucleon. One of the interesting additional spin contributions is from the strange quarks, called $\Delta s$. Although recent measurements show the static limits of the strange quark charge and magnetic contributions are consistent with zero [51], the nonzero value of $\Delta s$ is still under debate [52], because the weak coupling $(\propto(1-$ $\left.\left.4 \sin ^{2} \theta_{w}\right)\right)$ of $\Delta s$ with parity violating electron asymmetry does not allow a clear measurement of $\Delta s$ through electron scattering experiments.

However, $\Delta s$ also contributes to neutrino NCE scattering, as an axial vector isoscalar term, increasing the cross section for neutrino-proton NCE and decreasing the cross section for neutrino-neutron NCE. Figure 6 shows the ratio of $\nu p \rightarrow$ $\nu p$ to $v N \rightarrow v N$ candidates events, together with several predictions with nonzero $\Delta s$. Note MiniBooNE can only isolate neutrino-proton $\mathrm{NCE}$ in the case of high energy protons, and the denominator is chosen to be the total NCE events in order to cancel systematics. The fit to find $\Delta s$ is performed on this plot. After the fit, the best fit value of $\Delta s=0.08 \pm 0.26$ is found. Unfortunately, MiniBooNE does not have enough sensitivity to definitively determine nonzero $\Delta s$. This is due to the poor experimental proton-neutron separation which is only possible at high energy with large systematics. Therefore, a detector which has the ability to identify low energy protons, such as MicroBooNE [53], will have better sensitivity to $\Delta s$.

\section{MiniBooNE Oscillation Results}

The most well-known BSM search performed by the MiniBooNE experiment was for neutrino oscillations consistent with LSND. These are also the most thoroughly reviewed results. Here, we briefly describe the studies. We recommend [54] for a more extended discussion.

MiniBooNE was conceived in 1998, shortly after the LSND results had reached $3.8 \sigma$ significance and before the three massive neutrino model for active-flavor oscillations $(\nu \mathrm{SM})$ had been well established. However, it was clear that if LSND was observing an oscillation signal, the associated squared mass splitting $\left(\Delta m_{\text {large }}^{2}\right)$ was more than an order of magnitude larger than other evidence for oscillations. In this circumstance, a complicated three-neutrino appearance probability can reduce to a more simple two-neutrino case for designs with $(1.27 L / E) \approx 1 / \Delta m_{\text {large }}^{2}$, such as MiniBooNE.

This approach assumes no $C P$ violation in the mixing matrix, and hence equal probabilities of neutrino and antineutrino oscillations. Leptonic $C P$ violation in the mixing matrix had been discussed by Wolfenstein in 1978 [55] as a natural analogy to the quark sector. However, by extension of that analogy, the assumption was that this effect, if it existed, would be very small. As a result, theoretical interest in 1998 was largely isolated to $C P$ violation. In retrospect, this approach was naive, but this made sense as the guiding principle for the MiniBooNE design at the time. The goal was to test a simple two-neutrino oscillation model with equal probabilities of neutrinos and antineutrinos, on the basis that this would be a good approximation if the underlying

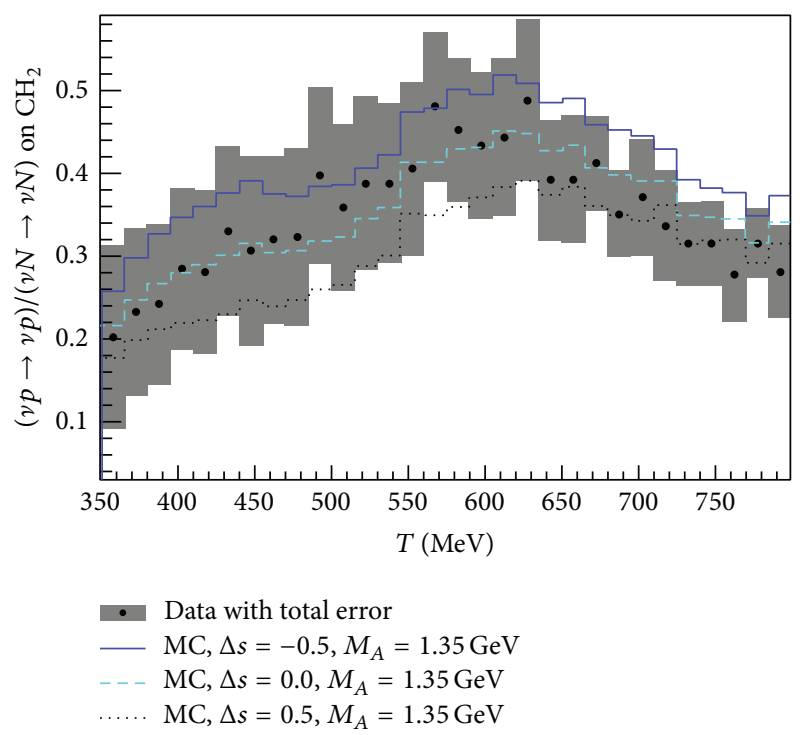

Figure 6: (Color online) The ratio of $v p \rightarrow v p$ to $v N \rightarrow v N$ as a function of the reconstructed total kinetic energy of nucleons [10].

reality was BSM physics. If a signal was not observed, the significantly different systematic errors were expected to result in a clear exclusion of the result. Thus, the MiniBooNE experiment began running in neutrino mode, which provided roughly $\sim 6$ times higher rate than antineutrino mode; a necessary choice since the MiniBooNE experiment was also relied on a significant Booster performance improvement. The results showed an anomalous excess of electron-like events in the $v_{\mu}$ dominant neutrino mode beam [56] that was similar to, but not in good agreement with, LSND. The experiment then switched to running in antineutrino mode, where a result in agreement with LSND was observed.

Rather than considering these events historically, we present both results together in the next section, followed by a discussion of interpretations and considerations of follow-up experiments. There is a world-wide effort to probe the sterile neutrino in the region $\Delta m^{2} \sim 1 \mathrm{eV}^{2}$ [57]. It is desirable for MiniBooNE to confirm this excess is electron-like, which is considered the sterile neutrino oscillation signal, not background gamma rays associated with $\nu_{\mu}\left(\bar{v}_{\mu}\right) \mathrm{NC}$ interactions. The MicroBooNE experiment [53] was proposed along this line. The MicroBooNE experiment features a large liquid argon (LAr) time projection chamber (TPC), and it has an ability to distinguish an electron (positron) and a gamma ray. The MicroBooNE experiment will start data taking in 2014. We will discuss more in a later section.

\subsection{The Neutrino and Antineutrino Appearance Oscillation} Results. After a decade of data collection, MiniBooNE's final appearance oscillation results have been published [58]. Figure 7 shows the electron candidate $\left(v_{\mu} \rightarrow v_{e}\right.$ oscillation candidate) distribution in neutrino mode and positron $\left(\bar{\nu}_{\mu} \rightarrow \bar{\nu}_{e}\right.$ oscillation candidate) distribution in antineutrino mode. Note that since the MiniBooNE detector is not magnetized, in general, it cannot distinguish between 

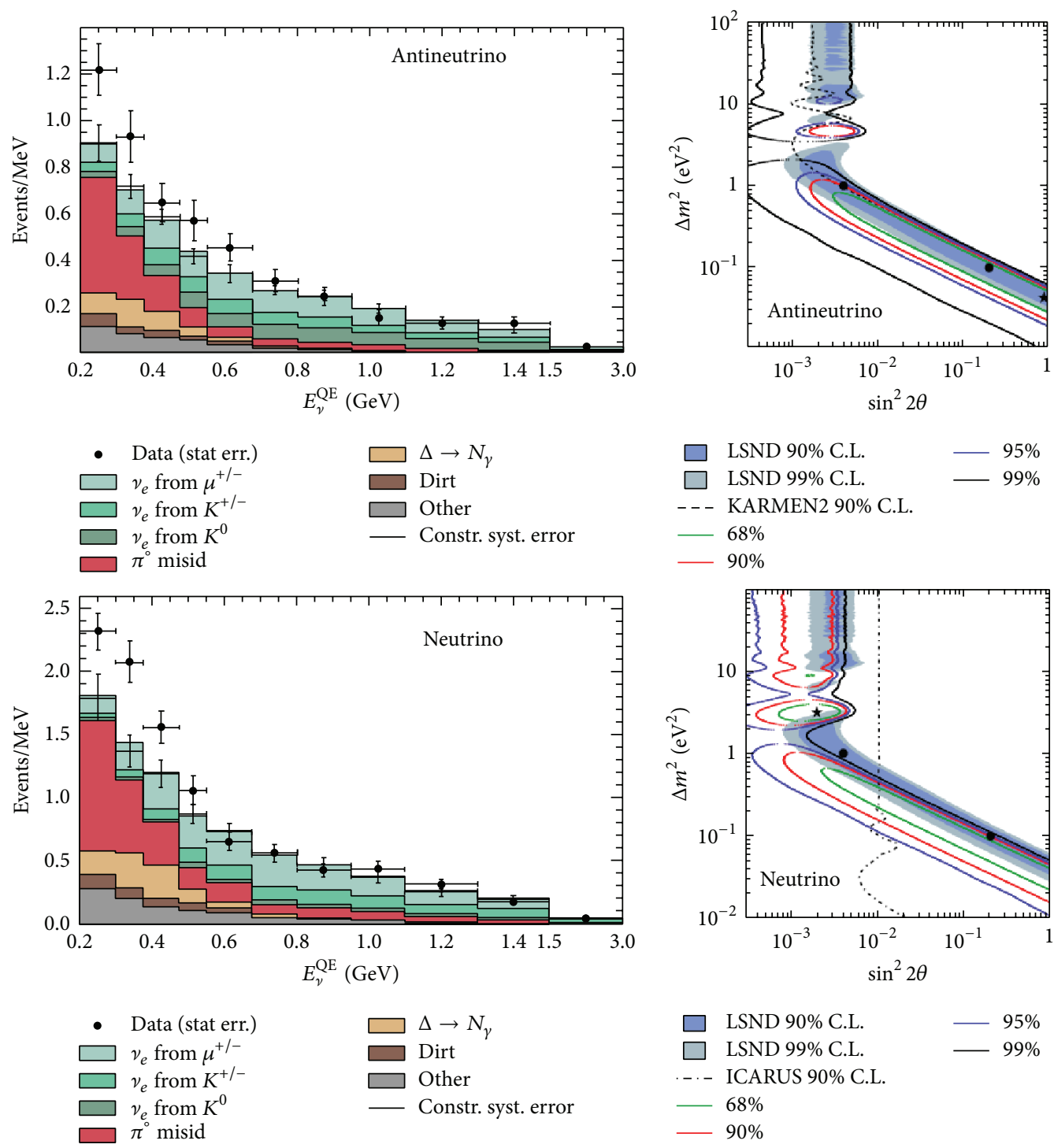

(a)

(b)

FIGURE 7: (Color online) The final MiniBooNE oscillation results [58]. (a) shows the reconstructed neutrino energy distribution of oscillation candidate events. The top is for antineutrino mode and the bottom is for neutrino mode. (b) shows the allowed region in $\Delta m^{2}-\sin ^{2} 2 \theta$, where the best fit points are shown in black stars.

electrons and positrons, and so both are grouped into the "electron-like" category.

MiniBooNE observed event excesses in both modes of running, but the results have slight qualitative difference. In neutrino mode (left bottom plot), there is a statistically significant $(3.8 \sigma)$ event excess in the low energy region. Although the excess is significant, the shape of the spectrum leaves some tension with the oscillation hypothesis from LSND, which you can see from the right bottom plot where the MiniBooNE best fit region does not overlap well with the LSND best fit region. MiniBooNE uses a likelihood-ratio technique [59], to find the best fit values $\left(\Delta m^{2}, \sin ^{2} 2 \theta\right)=$ $\left(3.14 \mathrm{eV}^{2}, 0.002\right)$ in neutrino mode, with $\chi^{2} /$ dof of $13.2 / 6.8$. In antineutrino mode (left top plot), the observed excess is not as statistically strong as neutrino mode $(2.8 \sigma)$. This is expected when one compares the protons on target in each mode and considers the lower antineutrino flux and cross section. Although the statistical significance is lower, shape agreement with the LSND hypothesis is better. Again this can be seen from the right top plot where the parameter space selected by the MiniBooNE data agrees with the LSND best fit region. The best fit point in this mode was $\left(0.05 \mathrm{eV}^{2}, 0.842\right)$ with $\chi^{2} /$ dof of 4.8/6.9.

The combined result significance is dominated by neutrino mode and is $3.8 \sigma$. It is possible to find compatible regions in a simple two-neutrino model between the two data sets [58]. However, we emphasize that considering MiniBooNE oscillations in the absence of other oscillation experiments leads to misunderstandings. We consider this point in a later section. 
4.1.1. Potential Nonoscillation Explanations. The backgroundonly $\chi^{2}$-probability for the MiniBooNE oscillation search was $1.6 \%$ and $0.5 \%$ relative to the best oscillation fits for neutrino and antineutrino mode, respectively. Nevertheless, it is important to explore in detail the potential SM explanations of the MiniBooNE results. In particular, a Cherenkov detector, such as MiniBooNE, lacks the ability to distinguish electrons from single photons. Therefore any single photon production mechanism via neutral current interactions is a likely suspect as a background to this search.

The primary source of single photons is the $\mathrm{NCl} \pi^{\circ}$ reaction, followed by $\pi^{\circ} \rightarrow \gamma \gamma$, where one photon is lost because it exits the detector or because the relativistic boost causes the energy to be too low to allow the Cherenkov signal to be identified. At the low energies of MiniBooNE, the background from two $\pi^{\circ}$ rings that merge is less important than the case where a photon is lost. Fortunately, MiniBooNE has the largest sample of well reconstructed $\mathrm{NC}^{\circ}$ events ever obtained. Keeping in mind that the largest uncertainties are in the production and not in the kinematics of the photons themselves, MiniBooNE was able to use this large data set to carefully evaluate this appearance background [60]. This study can constrain the variation of this largest misID background (red histogram in Figure 7(a)), and we have shown that if $\mathrm{NC} \pi^{\circ}$ was the source of the MiniBooNE excess, MiniBooNE's systematic error on the production would have to be underestimated by an order of magnitude [56]. This is not a likely solution to the problem, and so we turn to single photon production.

MiniBooNE also included the NC single photon process in their simulation. The process involves the single photon decay of a neutral current $\Delta$ resonance, which has a small but nonnegligible branching ratio $\left(<1 \%\right.$ of $\left.\mathrm{NCl} \pi^{\circ}\right)$. The rate of this process is strongly tied to the resonant production of pions; therefore MiniBooNE can utilize their in situ $\mathrm{NCl} \pi^{\circ}$ measurement to constrain this background. Therefore the variation of this second biggest misID background (light brown histograms in Figure 7(a)) is also constrained by the $\mathrm{NCl} \pi^{\circ}$ measurement, and we found this process was not large enough to explain the MiniBooNE excess [56].

After the first MiniBooNE oscillation result in 2007 [7], it was pointed out that there were additional single-photonproduction channels missing from the NUANCE [11] event simulation used by experiments such as MiniBooNE [61]. Figure 8 shows the relevant underlying diagram. This source, triangular anomaly mediated photon production, features weak coupling via the neutrino neutral current and strong coupling with nucleons or nuclei. In fact, a similar type of interaction was suggested originally in the 1980s [62]; however, it was not widely noted or further investigated. This type of process can generate a single gamma ray from a NC interaction. The strength of the anomaly mediated diagram was evaluated [63], and the event rate in MiniBooNE, after convoluting the BNB neutrino flux, was, at the time, estimated to be high enough to explain a part of the MiniBooNE excesses [64].

The initially high estimate, which may have explained the MiniBooNE result, led nuclear theorists to reevaluate

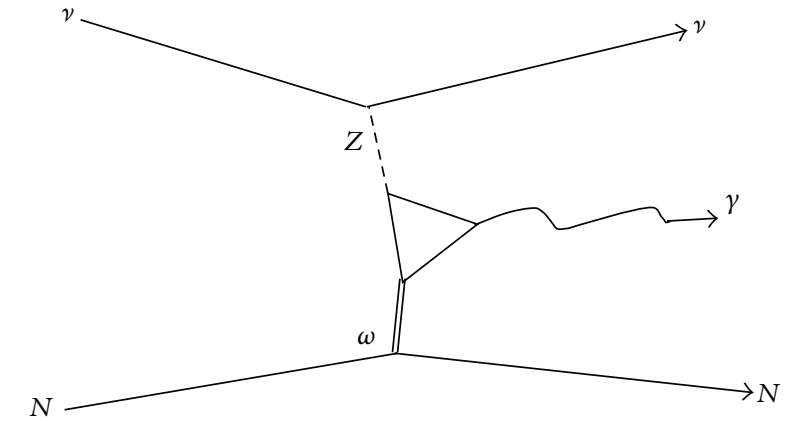

FIGURE 8: The triangular anomaly mediated photon production. The neutrino neutral current couples via Z-boson, and the target nucleon or nucleus couples with a strong force mediated vector meson, such as an omega meson.

this exotic " $Z-\gamma-\omega$ coupling," properly including nuclear effects, such as Pauli blocking and $\Delta$ resonance media width modification, as well as including careful calibrations of nuclear parameters from external data [65-67]. These are important to include since nuclear effects are sizable in this energy region. Note these nuclear effects tend to reduce the cross section.

Figure 9 shows our current knowledge of this channel [68]. The figure shows the total cross section of NC single photon production process per ${ }^{12} \mathrm{C}$ nucleus, which means the cross section includes all potential processes contributing to this final state topology ("0 muon +0 pion +1 photon $+N$ protons"), both incoherently (neutrino-nucleon interaction) and coherently (neutrino-nucleus interaction). As you see, all neutrino interaction generators used by experimentalists (GENIE [13], NEUT [14], and NUANCE [11]) tend to predict lower cross sections than state-of-the-art theoretical models by Wang et al. [65], Zhang and Serot [69], and Hill [64].

The NC single photon prediction may explain part of the excess, but it is not likely to explain all of it $[69,70]$. There was an active discussion on this channel at the recent INT workshop, and further experimental data on NC single photon production can help to guide more theoretical work [71].

Meanwhile, a BSM NC single photon model was proposed [72] where a decay of a heavy neutrino produces a single photon signal in the detector. Figure 10 shows the concept of such a model. The heavy neutrino is produced by the mixing with a muon neutrino; then the decay of the heavy neutrino leaves a photon signal in the detector. Interestingly, the required mass range of the heavy neutrino to produce such a signal in the MiniBooNE detector $\left(40 \mathrm{MeV}<m_{h}<\right.$ $80 \mathrm{MeV}$ ) is not constrained by other experiments. The beauty of this model is that it also explains the LSND signal, while evading the KARMEN null oscillation result [73].

At this time, NOMAD is the only experiment to have performed a dedicated NC single photon search [74]. The NOMAD result was consistent with its background prediction; thus, NOMAD set a limit on this channel. However, the limit was quoted with NOMAD's average energy $(<E\rangle$ $\sim 17 \mathrm{GeV}$ ) and is therefore not as relevant for lower energy experiments, such as MiniBooNE. Therefore, it is essential for 




FIgURE 9: (Color online) A comparison of the total cross section of $\mathrm{NC}$ photon production per ${ }^{12} \mathrm{C}$ nucleus [68]. The neutrino interaction generators used by experimentalists (GENIE [13], NEUT [14], and NUANCE [11]) tend to predict lower cross sections than stateof-the-art theoretical models (Wang et al. [65], Zhang and Serot [69], Hill [64]).



FIGURE 10: The concept of a heavy neutrino decay signal in the MiniBooNE detector [73]. The mixing of a neutrino with a hypothetical heavy neutrino and its short life time allows for it to decay in the MiniBooNE detector to leave a photon signal.

new experiments that seek to check the MiniBooNE results to have an ability to distinguish between electrons and photons, such as MicroBooNE [53].

4.1.2. Potential Oscillation Explanations. Numerous articles have been written on the potential of oscillation models to explain the MiniBooNE signal. In particular, we recommend [75] as a pedagogical discussion of the issues of fitting the data. We excerpt the results from this reference here.

When MiniBooNE and LSND results are considered within the context of the world's oscillation data, $\nu \mathrm{SM}$ is excluded, because a third mass splitting must be introduced. Because the $Z \rightarrow v \bar{v}$ results from LEP and SLD [1] limit the number of low mass active neutrinos to three, sterile neutrinos are introduced to allow for these data sets. Sterile neutrinos are a consequence of many theories and could evade limits from cosmology, as discussed in [57] (note recent Planck results [76] leave some tension with this interpretation).

If one sterile neutrino is added to the three active neutrinos, then the model is termed $(3+1)$. Two additional sterile neutrinos lead to a $(3+2)$ model and three result in a $(3+3)$ model. The mass states are mixtures of flavor states, and in these models, fits to the data yield mass states that are either mostly active flavors or mostly sterile flavors. The splitting between the mostly active and mostly sterile flavors is large, and the splittings between the active flavors are, comparatively, negligible. So, in sterile neutrino fits, the short-baseline approximation where the mostly active flavors are regarded as degenerate in mass is used. In such a model, $3+1$ models are simply two-neutrino models, such as what was initially proposed to explain LSND.

The disagreement between the MiniBooNE neutrino and antineutrino data leads to very poor fits for $3+1$ models. In order to introduce a difference in the neutrino oscillation probabilities, $C P$ violation must be included in the model. For the term which multiplies the CP-parameter to be significant, there must be two mass splittings that are within less than two orders of magnitude of each other. This can be accommodated in a $3+2$ model.

Since the MiniBooNE and LSND results were published, two new anomalies consistent with high $\Delta m^{2}$ oscillations were brought forward. These are the reactor anomaly [77], which has been interpreted as $\bar{\nu}_{e} \rightarrow \bar{\nu}_{s}$, and the gallium source anomaly [78] which can be interpreted as $\nu_{e} \rightarrow \nu_{s}$ [57]. Both anomalies have weaker significance than MiniBooNE and LSND, but they can be combined into a consistent model.

With this said, many experiments have searched for oscillations in the high $\Delta m^{2}$ region and found no evidence of oscillations. Reference [75] describes nine such results. The exclusion limits for electron-flavor disappearance and electron-flavor appearance can be shown to be compatible with the results of the four anomalous measurements. However, when muon-flavor disappearance is included, there is tension between the data sets which leads to low compatibility, except in the $3+3$ picture (or more elaborated version of $3+2$ model, called " $1+3+1$ " model [79]).

4.1.3. Near-Future Experiment Addressing the MiniBooNE Results. To test MiniBooNE signals in a model-independent way, a new experiment is planned on the BNB. The MicroBooNE experiment is a large liquid argon time projection chamber (LArTPC) experiment [53] at Fermilab, planning to start data taking from 2014. It is part of the US LArTPC program [80], with the eventual goal of an ultra-large LArTPC experiment, such as LBNE [81]. The experiments are motivated by the "bubble chamber level" LArTPC imaging quality.

Figure 11 shows a drawing [53] of MicroBooNE's 170 ton foam-insulated cryostat. The TPC volume is 89 tons. Ionized electrons along the neutrino-induced charged particle tracks are drifted via a high electric field in the TPC volume to the anode wires. The node wires are configured on three planes alternating by $60^{\circ}$ orientation, to allow 3-dimensional reconstruction of the tracks. The first 2 wire planes record 


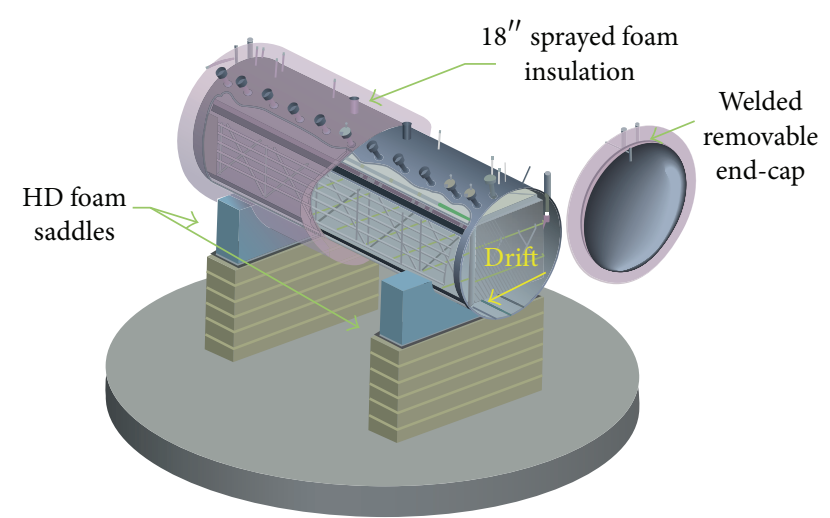

FIgURE 11: (Color online) A drawing of MicroBooNE cryostat [53]. The 170 ton cryostat contains the 89 ton TPC.

the signal from the induction on wires, and the last plane records the actual collection of ionization electrons.

An array of 8 -inch PMTs is equipped behind the wire planes [82]. The main purpose of this photon collection system is to reject out-of-time cosmic rays and to trigger on in-time signals, since the scintillation light from the interaction arrives in $\sim \mathrm{ns}$ whereas the time scale of ionization electron drift is of order $\sim$ ms. The detection of scintillation photons from LAr is not straightforward. First of all, the wavelength of Ar scintillation light is $128 \mathrm{~nm}$, which requires careful R\&D on potential wavelength shifters for use in LAr [83-85]. Second, the PMTs themselves behave differently in a cryogenic environment as compared to a warm environment, leading to the need for careful characterization [86].

The purity of the liquid argon must be kept very high to allow electrons to drift a long distance. Electronegative impurities (e.g., water and oxygen molecules) are removed through a custom made filter to achieve $\leq$ ppb level impurity $[87,88]$. Such filtering is also effective for removing nitrogen molecules, which do not affect electron drift but do attenuate scintillation light [89].

A high resolution LArTPC detector will be a powerful tool in understanding the MiniBooNE signal, because the detector is expected to have the excellent electron-photon separation. Energetic electrons and photons both produce an electromagnetic shower in a LArTPC. However, the initial $d E / d x$ of a single photon will be twice higher than in the single electron case in the first few centimeters before the track develops into the shower. Due to their high resolution capabilities, LArTPC detectors can distinguish this difference. Moreover, a displaced vertex, in the case of a photon conversion, can be distinguished from a track that is continuous from the vertex, indicative of an electron. The combination of these details can provide high efficiency background rejection for MicroBooNE.

\section{Test of Lorentz and CPT Violation}

Lorentz and CPT violation are scenarios motivated from Planck scale theories, such as string theory [90]. In the effective field theory framework, Lorentz violation contributes additional terms to the vacuum Lagrangian of neutrinos and hence modifies neutrino oscillations [91, 92]. Since Lorentz violating fields are of fixed direction in the universe, if Lorentz invariance is broken, the rotation of the Earth causes a sidereal time dependence of neutrino oscillation signals. There are number of phenomenological neutrino oscillation models based on Lorentz and CPT violation [93-95], some of which can explain the LSND excess [96]. In fact, a sidereal time dependence analysis of LSND data [97] failed to reject the Lorentz violation scenario. Therefore, it might be possible to reconcile LSND and MiniBooNE oscillation signals under Lorentz violation.

5.1. Analysis. Although Lorentz violation can be studied in any frame or coordinate system, it is convenient to choose one coordinate system to compare data sets. The standard choice is the Sun-centered celestial equatorial coordinates [98], where the origin of the coordinate is the center of the Sun. The orbital plane of the Earth is tilted so that the orbital axis and the rotation axis of the Earth align. This direction defines the $Z$-axis. The $X$-axis points vernal equinox, and the $Y$-axis is chosen to complete the right handed system. Because the time scale of the rotation of the galaxy is too long for any terrestrial experiments, the Sun-centered frame is the better choice to test rotation symmetry (by using the rotation of the Earth) and Lorentz boost (by using the revolution of the Earth).

Having defined the coordinates, one uses the standardmodel extension (SME) [99-101] as the framework for a general search for Lorentz violation. The SME can be considered a minimum extension of the SM, including the particle Lorentz and CPT violation. For the neutrino sector, the SME Lagrangian can be written as [91]

$$
\begin{aligned}
\mathscr{L}= & \frac{1}{2} i \bar{\psi}_{A} \Gamma_{A B}^{\mu} \stackrel{\leftrightarrow}{D_{\mu}} \psi_{B}-\bar{\psi}_{A} M_{A B} \psi_{B}+\text { h.c., } \\
\Gamma_{A B}^{\nu}= & \gamma^{\nu} \delta_{A B}+c_{A B}^{\mu \nu} \gamma_{\mu}+d_{A B}^{\mu \nu} \gamma_{5} \gamma_{\mu}+e_{A B}^{\nu} \\
& +i f_{A B}^{\nu} \gamma_{5}+\frac{1}{2} g_{A B}^{\lambda \mu \nu} \sigma_{\lambda \mu}, \\
M_{A B}= & m_{A B}+i m_{5 A B} \gamma_{5}+a_{A B}^{\mu} \gamma_{\mu}+b_{A B}^{\mu}+\frac{1}{2} H_{A B}^{\mu \nu} \sigma_{\mu \nu} .
\end{aligned}
$$

Here, the $A B$ subscripts represent the flavor basis. The first term of (7) and the first and second terms of (8) are the only nonzero terms in the SM, and the rest of the terms are from Lorentz violation.

The physics consequences predicted by Lorentz violation are very rich. Among them, we are interested in Lorentz violating neutrino oscillations. Neutrino oscillations are natural interferometers and they are sensitive to small effects such as Lorentz violation. The smoking gun of Lorentz violation is the sidereal time dependence of physics observables. Therefore, we used the Lorentz violating $\nu_{\mu} \rightarrow v_{e}\left(\bar{v}_{\mu} \rightarrow \bar{v}_{e}\right)$ neutrino oscillation formula derived from above Lagrangian [102] to fit the sidereal time distribution of the $\nu_{\mu} \rightarrow \nu_{e}\left(\bar{\nu}_{\mu} \rightarrow \bar{\nu}_{e}\right)$ oscillation candidate data. Here, potentially, any day-night effect, either from the beam or from the detector, could mimic 




(a)

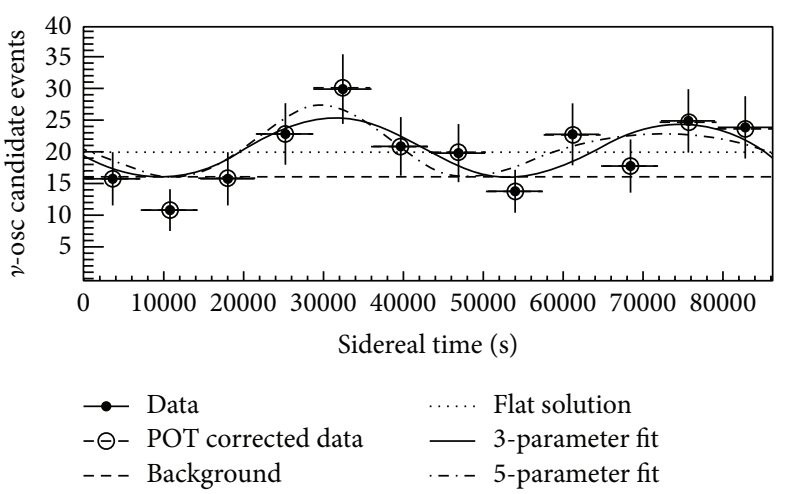

(b)

Figure 12: The MiniBooNE Lorentz violation results [103]. (a) shows the neutrino mode electron-like low energy excess sidereal time distribution, and (b) shows the antineutrino mode sidereal time distribution. Here, the data with a POT correction (open circle) show the size of the beam day-night variation. There are three fit curves based on different assumptions, a flat solution (dotted), a three-parameter fit (solid curve), and a full five-parameter fit (dash-dotted curve).

the sidereal time distribution. MiniBooNE studied effects versus the time distribution of the delivered POT and the high statistics $\nu_{\mu}\left(\bar{\nu}_{\mu}\right)$ CCQE sample $[18,36]$ and confirmed that day-night effects on both $\nu_{e}$ and $\bar{\nu}_{e}$ oscillation candidates are well below statistical errors.

5.2. Results. Figure 12 shows the neutrino and antineutrino mode electron-like events as a function of sidereal time [103]. Since background events are time-independent, we fit curves on the flat time-independent background (dashed lines). There are three curves fit to the data, depending on different hypotheses. A flat solution (dotted lines) assumes only timeindependent Lorentz violating term, a 3-parameter fit (solid lines) includes all CPT-odd Lorentz violating terms, and a 5-parameter fit (dash-dotted lines) is the full parameter fit including both CPT-odd and CPT-even Lorentz violating terms. Although the antineutrino mode electron-like events show a rather interesting sidereal time dependence, the statistical significance is still low. Therefore, MiniBooNE found that the data are consistent with no Lorentz violation. This analysis provided the first limits on five time independent SME coefficients, at the level of $10^{-20} \mathrm{GeV}$ (CPT-odd) and order $10^{-20}$ (CPT-even). Further analysis inferred limits on each SME coefficient, and, together with limits from the MINOS near detector [104, 105], it turns out these limits leave tension to reconcile the MiniBooNE and LSND data sets under a simple Lorentz violation motivated scenario [4].

In fact, existing limits from MiniBooNE [103], MINOS [104-107], IceCube [108], and Double Chooz [109, 110] set very tight limits on possible Lorentz violation in the neutrino sector at the terrestrial level. This was one of the reasons why the superluminal neutrino signal from OPERA [111] was suspicious from the beginning. Such a signal would have required very large Lorentz violation, while avoiding all these constraints when writing down the theory. Strictly speaking, limits on Lorentz violation from the oscillation experiments cannot be applied directly to the neutrino time of flight (TOF) measurement [112]. However, introducing

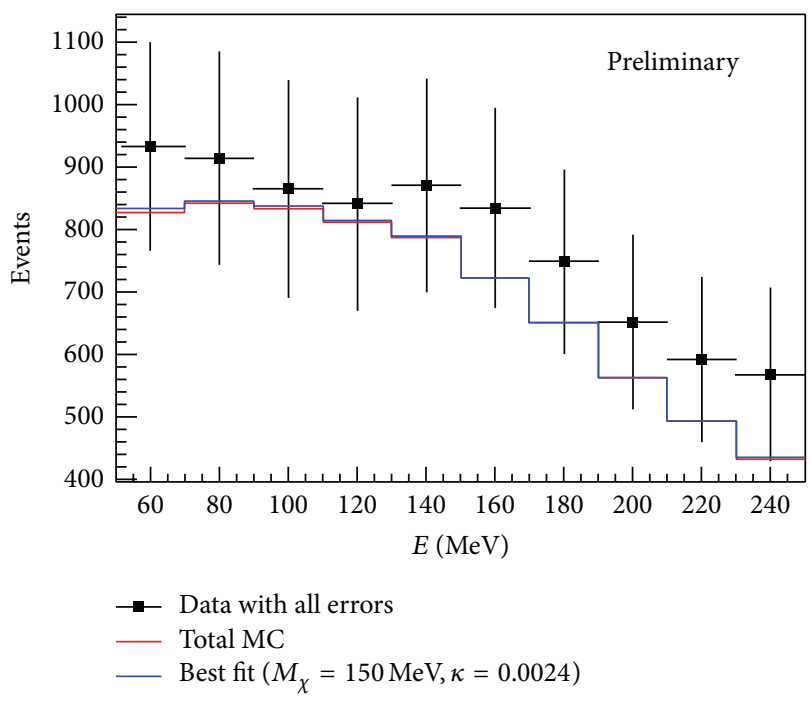

FIGURE 13: (Color online) The dark matter fit result to the NCE data [116].

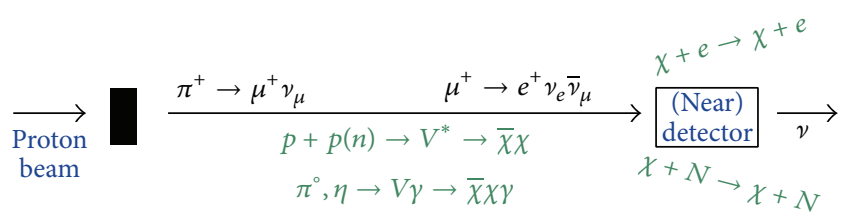

FIgure 14: (Color online) The concept of the dark matter beam in MiniBooNE [116]. The dominant production mode of dark matter particles is decays of the mediator particles created by decays of neutral mesons. The dark matter particles can be also made through the direct collisions of protons on the beam dump.

large Lorentz violation in the neutrino TOF without other large parameters such as those associated with oscillations seems unnatural. 


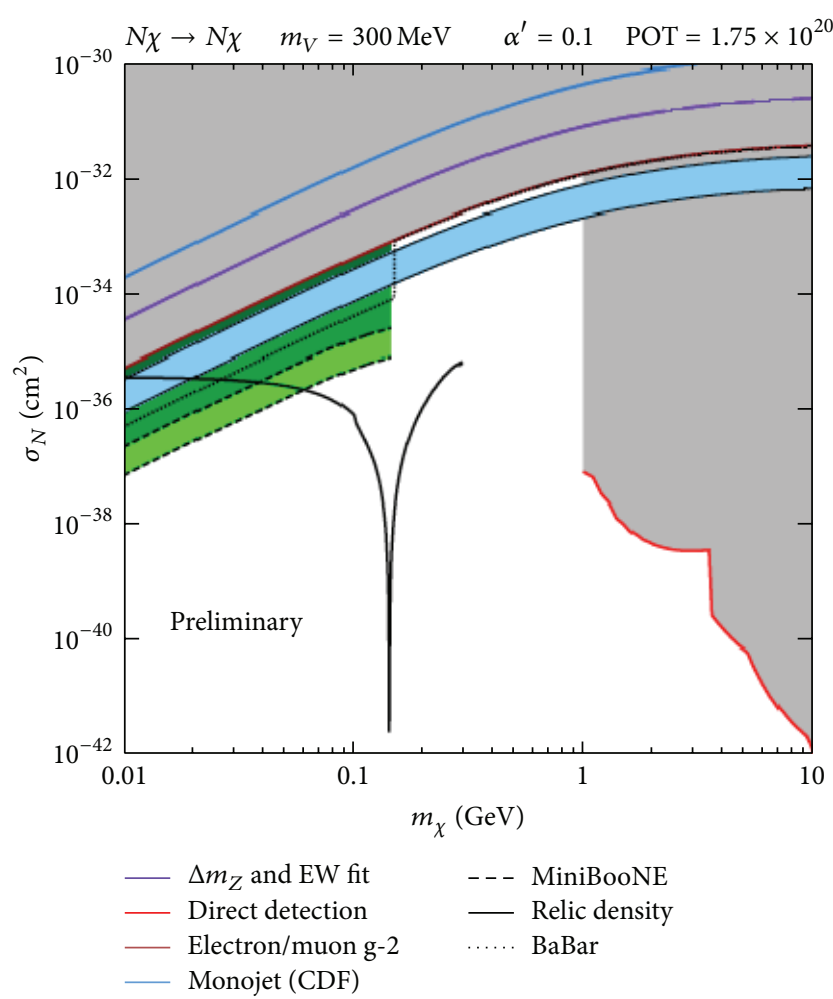

(a)

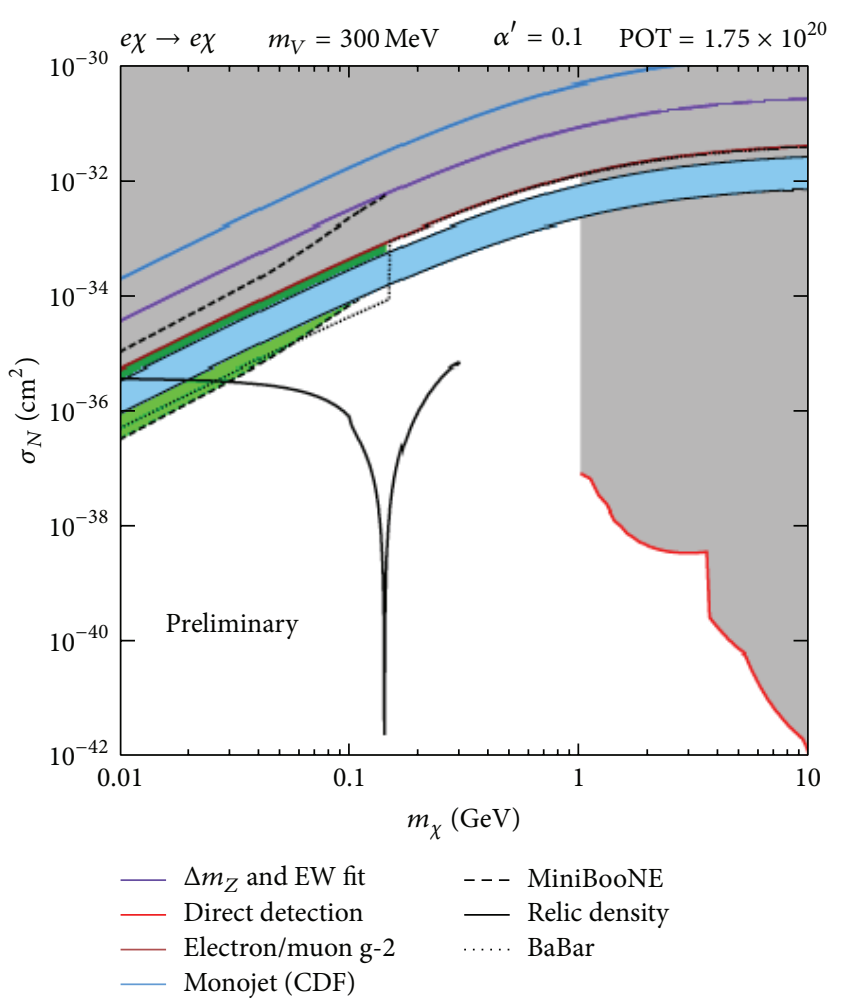

(b)

Figure 15: (color online) The MiniBooNE dark matter particles search phase space [116]. Here, the $x$-axis is the dark matter mass $m_{\chi}$, and the $y$-axis is either the dark matter-nucleon or dark matter-electron cross section, assuming the vector mediator mass and the gauge coupling $\left(m_{V}=300 \mathrm{MeV}\right.$ and $\left.\alpha=0.1\right)$. The MiniBooNE exclusion region can be seen in green.

\section{Dark Matter Search}

The proton collisions on target in the BNB line that produce a large flux of neutrinos could, potentially, produce sub-GeV scale dark matter particles that mimic NCE interactions in the MiniBooNE detector [113-115]. The most interesting scenario is that this light dark matter particle is the dark matter of the universe, which requires a light vector mediator particle (called a "dark photon"), in the model in order to obtain an efficient annihilation cross section. The minimum extension of the SM with the light dark matter particle and the vector mediator can be written in the following way [114]:

$$
\begin{aligned}
\mathscr{L}= & \mathscr{L}_{S M}-\frac{1}{4} V_{\mu \nu}^{2}+\frac{1}{2} m_{V}^{2} V_{\mu}^{2}+\kappa V_{\nu} \partial_{\mu} F^{\mu \nu} \\
& +\left|D_{\mu} \chi\right|^{2}-m_{\chi}^{2}|\chi|^{2}+\cdots
\end{aligned}
$$

The model has four free parameters: the mass of the light dark matter $m_{\chi}$, the mass of the vector mediator $m_{V}$, kinetic mixing of the vector mediator and the photon $\kappa$, and the vector mediator's gauge coupling $e^{\prime}$ ( or $\alpha^{\prime}=e^{\prime 2} / 4 \pi$ ). Nonzero $\kappa$ leads to the decay of neutral mesons to a photon and a dark photon, and the dark photon in turn can decay to dark matter particles. This would be the dominant process to produce dark matter particles in the BNB. The second process is direct production from the parton level annihilation by protons colliding in the target.

6.1. MiniBooNE Searches for Dark Matter Particles. MiniBooNE tested this model with the existing antineutrino NCE data set, taken during the oscillation studies. Figure 13 shows the fit result with a light dark matter particles hypothesis [116]. The plot shows the total energy distribution of the antineutrino NCE sample, and the red and blue histograms show before and after the fit. The best fit values are $M_{\chi}=$ $150 \mathrm{MeV}$ and $\kappa=0.0024$. As can be seen, the current sensitivity to the light dark matter model is low.

The antineutrino mode data set is used because it has a lower neutrino interaction rate than the neutrino mode beam. Nevertheless, due to the antineutrino backgrounds, only weak limits are obtained on the kinetic mixing parameter $\kappa$.

This motivated a tuning of the proton beam that allowed MiniBooNE to run in a mode in which the protons are directed onto the beam dump instead of the target, eliminating the DIF neutrino flux. Figure 14 shows the schematic of this measurement [116]. The beam-dump mode is achieved by tuning the $\sim 1 \mathrm{~mm}$ beam to aim $0.9 \mathrm{~cm}$ gap between the beryllium target rod and the inner conductor of the horn, to hit the beam dump located at the end of decay pipe 

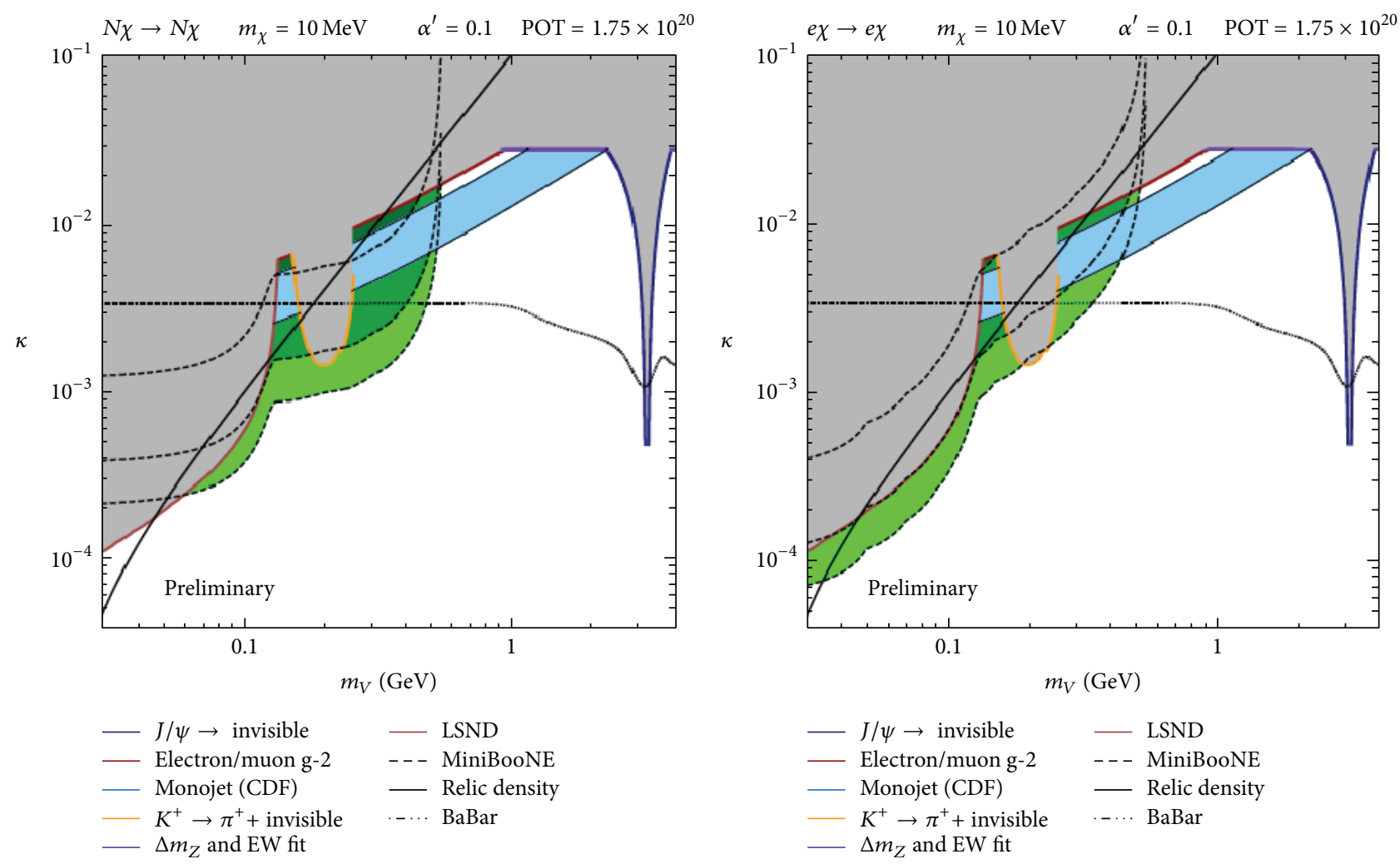

Figure 16: (Color online) The MiniBooNE dark matter search phase space [116]. Here, $x$-axis is the vector mediator mass $m_{V}$, and the $y$-axis is the kinetic mixing parameter $\kappa$, assuming the dark matter mass and the gauge coupling $\left(m_{\chi}=10 \mathrm{MeV}\right.$ and $\left.\alpha=0.1\right)$. MiniBooNE exclusion region can be seen in green.

(50 $\mathrm{m}$ from the target) directly. This reduces the neutrino background by roughly a factor of 67. Dark matter production is largely unaffected in this run mode since it occurs through neutral meson decay. MiniBooNE is now running in this configuration. The goal is to accumulate $1.75 \times 10^{20}$ POT data before MicroBooNE starts beam data taking in the neutrino mode, not the beam-dump mode.

\subsection{Parameter Space of Light Dark Matter Particles and Vector} Mediators. Figure 15 shows the two-dimensional phase space of dark matter-nucleon and dark matter-electron scattering cross sections versus dark matter mass $m_{\chi}$ [116]. The limits from direct searches end up at the right side $\left(m_{\chi} \sim 1 \mathrm{GeV}\right)$, and the left-side light dark matter region is explored by other techniques, such as rare decays and collider physics. MiniBooNE addresses direct light dark matter searches. In the case of either interaction, MiniBooNE is sensitive to the dark matter mass in the 10 to $200 \mathrm{MeV}$ mass region.

There are many reasons why such a light dark matter search is interesting. First, recent data [117-120] from the direct WIMP (weakly interacting massive particle) searches suggest possible signals of dark matter particles in the lighter mass region. For example, SuperCDMS is also aiming the low mass dark matter search by utilizing the ionization signals [121]. Second, the muon g-2 anomaly can be explained by the presence of a vector mediator $[122,123]$. Although the interesting phase space of muon $\mathrm{g}-2$ was already excluded by other experiments, MiniBooNE can further push the limits in this region.

The sensitivity that is obtained from the dark matterelectron scattering looks weaker than dark matter-nucleon in the $\sigma-m_{\chi}$ phase space (Figure 15(b)); however, as Figure 16 shows, the limit from the dark matter-electron interaction can be stronger in the low vector mass region in $\kappa-m_{V}$ phase space [116]. Therefore, both channels are complimentary and MiniBooNE should strive to measure both. There was a little interest in $v$-e elastic scattering because of its small cross section, but this electron channel is as important as the nucleon channel for the dark matter search.

6.3. Dark Matter Time of Flight (TOF). MiniBooNE's sensitivity to dark matter particles can be further improved by combining event topology and kinematics with the timing information. Figure 17 shows the "dark matter TOF" concept. The dark matter particles are most likely produced at the beam dump after prompt decays of neutral pions or etas $\left(<10^{-16} \mathrm{sec}\right)$, so the dark matter production is localized in both time and space. This would result in a dark matter beam that has a well-defined timing and allows us to perform the TOF-based searches. The heavier dark matter particles should be slower than the neutrinos (as well as the speed of light). Thus the dark matter particles would lag behind the bunch center and separate from the neutrino background. 


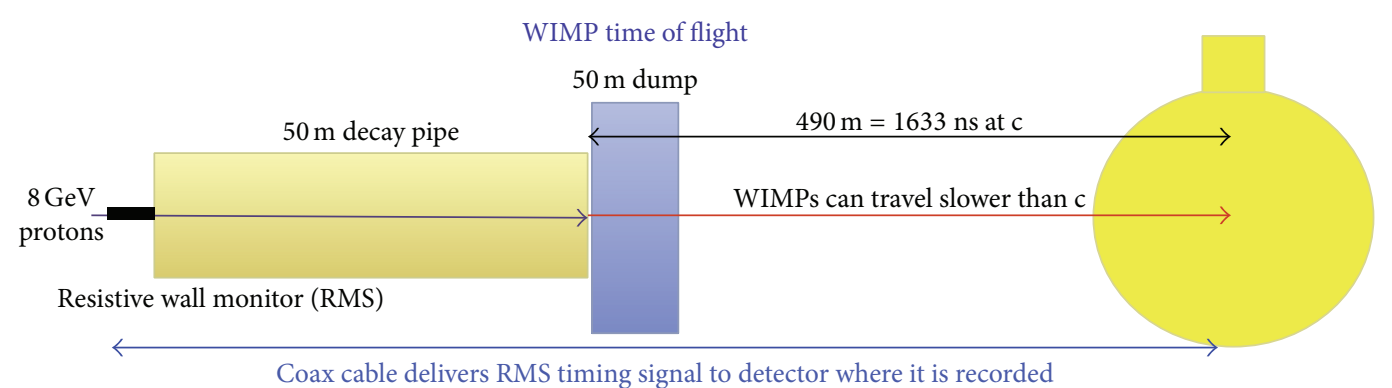

FIGURE 17: (Color online) The concept of dark matter particles TOF. Because of the localization of the dark matter particle production in time and in space, the dark matter beam has well-defined timing structure.

In the Fermilab Booster, the 81 bunches have 19 ns separations (Section 2.1). MiniBooNE defines events within $4 \mathrm{~ns}<$ $T<16 \mathrm{~ns}$ from the bunch center as the in-time events, and the $T<4$ ns and $T>16$ ns events are out-time. The absolute timing information of all bunches is recorded by the resistive wall monitor (RWM) which is located just before the target. Using the previous MiniBooNE antineutrino run to test this idea, Figure 18 shows the overlaid profile of all bunches of antineutrino NCE candidate events [116]. As expected, the data shows the peak in in-time region, because the data is dominated by antineutrino NCE interactions.

A beam-dump test run was performed for one week during 2012 running. During the beam-dump mode test run, the timing of neutrinos was tested using CC interaction. Since the CC interaction is detected through the prompt Cherenkov light from the muons, timing resolution is better than NCE events. Using the new system installed for the beamdump run, MiniBooNE achieved $1.5 \mathrm{~ns}$ resolution [116]. The resolution will be worse for NCE because of the nature of the exponential decay of scintillation light, but MiniBooNE, nevertheless, still expects $\sim 4 \mathrm{~ns}$ resolutions. This gives full confidence for MiniBooNE to perform a full beam-dump run.

\section{Conclusion}

Since beginning its run in 2002, MiniBooNE has been searching for new physics in a wide variety of ways. The most important results have been those related to oscillations of sterile neutrinos, which has pushed the community toward new and exciting experiments in the future [53, 57, 124-126]. MiniBooNE also tested for possible signals from the Planck scales, and set very strong constraints on Lorentz violation. MiniBooNE's light dark matter search with a beam-dump configuration run is a unique opportunity that can provide the best limit on the dark matter mass in the 10 to $200 \mathrm{MeV}$ range. All of these searches have been grounded in the revolutionary set of cross section measurements performed with MiniBooNE. This experiment demonstrates the rich possibilities to go beyond the standard model in low cost short-baseline venues and encourages a strong investment in future programs.

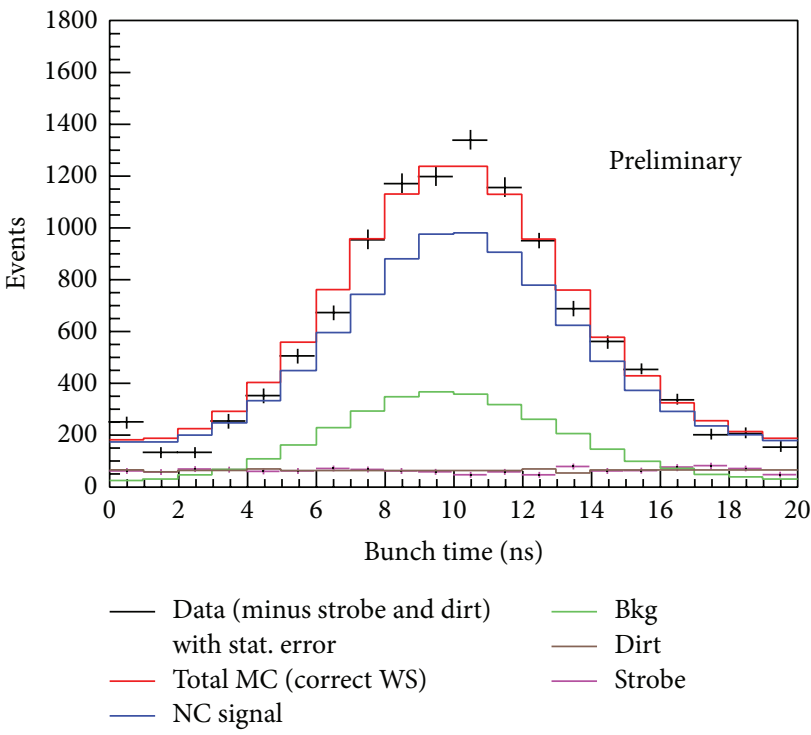

FIGURE 18: (Color online) The reconstructed NCE event time profile for the antineutrino mode beam. The events are overlaid relative to the bunch center. As expected, the data peaks in the bunch center, which means these are dominated with antineutrino interactions and there is no delay of events.

\section{Conflict of Interests}

The authors declare that there is no conflict of interests regarding the publication of this paper.

\section{Acknowledgments}

Janet Conrad thanks the National Science Foundation for support through NSF-PHY-1205175. The authors thank Brian Batell for inputs about light dark matter physics; also they thank Joshua Spitz for careful reading of the paper and valuable comments.

\section{References}

[1] J. Beringer, J.-F. Arguin, R. M. Barnett et al., "Review of particle physics," Physical Review D, vol. 86, Article ID 010001, 2012. 
[2] K. Abe, J. Adam, H. Aihara et al., "Observation of electron neutrino appearance in a muon neutrino beam," Physical Review Letters, vol. 112, no. 6, Article ID 061802, 8 pages, 2014.

[3] A. Aguilar-Arevalo, L. B. Auerbach, R. L. Burman et al., "Evidence for neutrino oscillations from the observation of electron anti-neutrinos in a muon anti-neutrino beam," Physical Review D, vol. 64, Article ID 112007, 2001.

[4] T. Katori, "Tests of Lorentz and CPT violation with MiniBooNE neutrino oscillation excesses," Modern Physics Letters A, vol. 27, no. 25, Article ID 1230024, 2012.

[5] A. Aguilar-Arevalo, C. E. Anderson, A. O. Bazarko et al., "Neutrino flux prediction at MiniBooNE" Physical Review D, vol. 79, Article ID 072002, 2009.

[6] A. Aguilar-Arevalo, C. E. Andersonp, L. M. Bartoszekg et al., "The MiniBooNE detector," Nuclear Instruments and Methods in Physics Research Section A, vol. 599, pp. 28-46, 2009.

[7] A. Aguilar-Arevalo, A. O. Bazarko, S. J. Brice et al., "Search for electron neutrino appearance at the $\Delta m^{2} \sim 1 \mathrm{eV}^{2}$ scale," Physical Review Letters, vol. 98, Article ID 231801, 2007.

[8] A. A. Aguilar-Arevalo, C. E. Anderson, A. O. Bazarko et al., "Measurement of neutrino-induced charged-current charged pion production cross sections on mineral oil at $E_{\gamma} \sim 1 \mathrm{GeV}$," Physical Review D, vol. 83, no. 5, Article ID 052007, 2011.

[9] A. Aguilar-Arevalo, C. E. Anderson, A. O. Bazarko et al., "Measurement of $v_{\mu}$-induced charged-current neutral pion production cross sections on mineral oil at $\mathbf{E}_{\mathbf{v}} \in 0.5-2.0 \mathrm{GeV}$," Physical Review D, vol. 83, Article ID 052009, 2011.

[10] A. Aguilar-Arevalo, B. G. Tice, [MiniBooNE collaboration] et al., "Measurement of the neutrino neutral-current elastic differential cross section on mineral oil at $E_{v} \sim 1 \mathrm{GeV}$," Physical Review D, vol. 82, Article ID 092005, 2010.

[11] D. Casper, "The nuance neutrino simulation, and the future," Nuclear Physics B, vol. 112, no. 1-3, pp. 161-170, 2002.

[12] C. Juszczak, "Running nuwro," Acta Physica Polonica B, vol. 40, pp. 2507-2512, 2009.

[13] C. Andreopoulos, A. Bell, D. Bhattacharya et al., "The GENIE neutrino Monte Carlo generator," Nuclear Instruments and Methods in Physics Research Section A, vol. 614, pp. 87-104, 2010.

[14] Y. Hayato, "A neutrino interaction simulation program library NEUT," Acta Physica Polonica B, vol. 40, pp. 2477-2489, 2009.

[15] O. Buss, T. Gaitanos, K. Gallmeister et al., "Transporttheoretical description of nuclear reactions," Physics Reports, vol. 512, no. 1-2, pp. 1-124, 2012.

[16] C. Juszczak, J. T. Sobczyk, and J. Zmuda, "Extraction of the axial mass parameter from MiniBooNE neutrino quasielastic double differential cross-section data," Physical Review C, vol. 82, Article ID 045502, 2010.

[17] A. Aguilar-Arevalo, A. O. Bazarko, S. J. Brice et al., "Measurement of muon neutrino quasielastic scattering on carbon," Physical Review Letters, vol. 100, Article ID 032301, 2008.

[18] A. Aguilar-Arevalo, C. E. Anderson, A. O. Bazarko et al., "First measurement of the muon neutrino charged current quasielastic double differential cross section," Physical Review D, vol. 81, no. 9, Article ID 092005, 22 pages, 2010.

[19] M. Martini, M. Ericson, G. Chanfray, and J. Marteau, "A unified approach for nucleon knock-out, coherent and incoherent pion production in neutrino interactions with nuclei," Physical Review C, vol. 80, Article ID 065501, 2009.

[20] J. Nieves, I. R. Simo, and M. V. Vacas, "Inclusive charged-current neutrino-nucleus reactions," Physical Review C, vol. 83, no. 4, Article ID 045501, 2011.
[21] M. Martini, M. Ericson, and G. Chanfray, "Neutrino quasielastic interaction and nuclear dynamics," Physical Review C, vol. 84, no. 5, Article ID 055502, 2011.

[22] J. Nieves, I. R. Simo, and M. V. Vacas, "The nucleon axial mass and the MiniBooNE quasielastic neutrino-nucleus scattering problem," Physics Letters B, vol. 707, no. 1, pp. 72-75, 2012.

[23] J. Amaro, M. Barbaro, J. Caballero, T. Donnelly, and C. Williamson, "Meson-exchange currents and quasielastic neutrino cross sections in the superscaling approximation model," Physics Letters B, vol. 696, pp. 151-155, 2011.

[24] A. Bodek, H. Budd, and M. Christy, "Neutrino quasielastic scattering on nuclear targets," The European Physical Journal C, vol. 71, article 1726, 2011.

[25] A. Meucci, C. Giusti, and F. D. Pacati, "Relativistic descriptions of final-state interactions in neutral-current neutrino-nucleus scattering at MiniBooNE kinematicsc," Physical Review D, vol. 84, Article ID 113003, 2011.

[26] O. Lalakulich, K. Gallmeister, and U. Mosel, "Complete set of polarization transfer observables for the ${ }^{16} \mathrm{O}(\underset{p}{\vec{p}}, \underset{p}{\vec{p}})^{16} \mathrm{~F}$ reaction at $296 \mathrm{MeV}$ and 0," Physical Review C, vol. 84, no. 1, Article ID 014614, 8 pages, 2012.

[27] G. Fiorentini, D. W. Schmitz, and P. A. Rodrigues, "Measurement of muon neutrino quasielastic scattering on a hydrocarbon target at $E_{v} \sim 3.5 \mathrm{GeV}$," Physical Review Letters, vol. 111, Article ID 022502, 2013.

[28] L. Fields, J. Chvojka, L. Aliaga et al., "Measurement of muon antineutrino Quasi-elastic scattering on a hydrocarbon target at E_ $\nu \sim 3.5 \mathrm{GeV}$," Physical Review Letters, vol. 111, Article ID 022501, 2013.

[29] K. Abe, N. Abgrall, H. Aihara et al., "Measurement of the inclusive $v_{\mu}$ charged current cross section on carbon in the near detector of the T2K experiment," Physical Review D, vol. 87, Article ID 092003, 2013.

[30] M. Martini, M. Ericson, G. Chanfray, and J. Marteau, "Neutrino and antineutrino quasielastic interactions with nuclei," Physical Review C, vol. 81, Article ID 045502, 2010.

[31] M. Martini and M. Ericson, "Quasielastic and multinucleon excitations in antineutrino-nucleus interactions," Physical Review C, vol. 87, no. 6, Article ID 065501, 2013.

[32] J. Nieves, I. Ruiz Simo, and M. Vicente Vacas, “Two particle-hole excitations in charged current quasielastic antineutrino-nucleus scattering," Physics Letters B, vol. 721, pp. 90-93, 2013.

[33] A. Meucci and C. Giusti, "Relativistic descriptions of finalstate interactions in charged-current quasielastic antineutrinonucleus scattering at MiniBooNE kinematics," Physical Review $D$, vol. 85, Article ID 093002, 2010.

[34] J. Amaro, M. Barbaro, J. Caballero, and T. Donnelly, "Mesonexchange currents and quasielastic antineutrino cross sections in the superscaling approximation," Physical Review Letters, vol. 108, Article ID 152501, 2012.

[35] A. A. Aguilar-Arevalo, C. E. Anderson, S. J. Brice et al., "Measurement of the neutrino component of an antineutrino beam observed by a nonmagnetized detector," Physical Review D, vol. 84, no. 7, Article ID 072005, 2011.

[36] A. Aguilar-Arevalo, B. C. Brown, L. Bugel et al., "First measurement of the muon antineutrino double-differential chargedcurrent quasielastic cross section," Physical Review D, vol. 88, Article ID 032001, 2013.

[37] J. Grange and R. Dharmapalan, "New anti-neutrino crosssection results from MiniBooNE," http://arxiv.org/abs/1304 .7395 . 
[38] M. Martini, M. Ericson, and G. Chanfray, "Neutrino energy reconstruction problems and neutrino oscillations," Physical Review D, vol. 85, Article ID 093012, 2012.

[39] D. Meloni and M. Martini, "Revisiting the T2K data using different models for the neutrino-nucleus cross sections," Physics Letters B, vol. 716, no. 1, pp. 186-192, 2012.

[40] J. Nieves, F. Sanchez, I. Ruiz Simo, and M. Vicente Vacas, "Neutrino energy reconstruction and the shape of the CCQElike total cross section," Physical Review D, vol. 85, Article ID 113008, 2012.

[41] O. Lalakulich, U. Mosel, and K. Gallmeister, "Neutrino energy reconstruction in quasielastic-like scattering in the MiniBooNE and T2K experiments," Physical Review C, vol. 86, Article ID 054606, 2012.

[42] A. A. Aguilar-Arevalo, C. E. Anderson, A. O. Bazarko et al., "Measurement of $\nu_{\mu}$ and $\bar{\nu}_{\mu}$ induced neutral current single $\pi^{0}$ production cross sections on mineral oil at $E_{v} \sim \mathcal{O}(1 \mathrm{GeV})$," Physical Review D, vol. 81, Article ID 013005, 2010.

[43] O. Lalakulich and U. Mosel, "Pion production in the MiniBooNE experiment," Physical Review C, vol. 87, no. 1, Article ID 014602, 2013.

[44] E. Hernndez, J. Nieves, and M. J. V. Vacas, "Single pion production in neutrino nucleus scattering," Physical Review D, vol. 87, Article ID 113009, 2013.

[45] A. Aguilar-Arevalo, B. C. Brown, L. Bugel et al., "Measurement of the antineutrino neutral-current elastic differential cross section," http://arxiv.org/abs/arXiv:1309.7257.

[46] T. Leitner, L. Alvarez-Ruso, and U. Mosel, "Neutral current neutrino-nucleus interactions at intermediate energies," Physical Review C, vol. 74, Article ID 065502, 2006.

[47] J. R. Ellis, K. A. Olive, and C. Savage, "Hadronic uncertainties in the elastic scattering of supersymmetric dark matter," Physical Review D, vol. 77, Article ID 065026, 2008.

[48] J. Ashmana, B. Badelekb, G. Baum et al., "A measurement of the spin asymmetry and determination of the structure function $g_{1}$ in deep inelastic muon-proton scattering," Physics Letters B, vol. 206, no. 2, pp. 364-370, 1988.

[49] D. Adams, B. Adeva, E. Arik et al., "Measurement of the spindependent structure function $g_{1}(x)$ of the proton," Physics Letters B, vol. 329, pp. 399-406, 1994.

[50] V. W. Hughes, V. Papavassiliou, R. Piegaia, K. P. Schüler, and G. Baum, "The integral of the spin-dependent structure function glp and the Ellis-Jaffe sum rule," Physics Letters B, vol. 212, no. 4, pp. 511-514, 1988.

[51] D. Androić, D. S. Armstrong, J. Arvieux et al., "Strange quark contributions to parity-violating asymmetries in the backward angle G0 electron scattering experiment," Physical Review Letters, vol. 104, Article ID 012001, 2010.

[52] S. F. Pate, D. W. McKee, and V. Papavassiliou, "Strange quark contribution to the vector and axial form factors of the nucleon: combined analysis of data from the G0, HAPPEx, and Brookhaven E734 experiments," Physical Review C, vol. 78, Article ID 015207, 2008.

[53] L. Camilleri, "MicroBooNE," Nuclear Physics B-Proceedings Supplements, vol. 237-238, pp. 181-183, 2013.

[54] J. M. Conrad, W. C. Louis, and M. H. Shaevitz, "The LSND and MiniBooNE oscillation searches at high $\Delta m^{2}$," Annual Review of Nuclear and Particle Science, vol. 63, pp. 45-67, 2013.

[55] L. Wolfenstein, "Oscillations among three neutrino types and CP violation,” Physical Review D, vol. 18, no. 3, pp. 958-960, 1978.
[56] A. Aguilar-Arevalo, B. C. Brown, L. Bugel et al., "Unexplained excess of electronlike events from a $1-\mathrm{GeV}$ neutrino beam," Physical Review Letters, vol. 102, Article ID 101802, 2009.

[57] K. N. Abazajian, M. A. Acero, S. K. Agarwalla et al., "Light sterile neutrinos: a white paper," http://arxiv.org/abs/1204.5379.

[58] A. Aguilar-Arevalo, B. C. Brown, L. Bugel et al., "Improved search for $\bar{\nu}_{\mu} \rightarrow \bar{\nu}_{e}$ oscillations in the MiniBooNE experiment," Physical Review Letters, vol. 110, no. 16, Article ID 161801, 2013.

[59] A. Aguilar-Arevalo, C. Anderson, S. Brice et al., "Event excess in the MiniBooNE search for $\bar{v}_{\mu} \rightarrow \bar{v}_{e}$ oscillations," Physical Review Letters, vol. 105, Article ID 181801, 2010.

[60] A. Aguilar-Arevalo, C. E. Anderson, A. O. Bazarko et al., "First observation of coherent $\pi^{0}$ production in neutrino-nucleus interactions with $E_{v}<2 \mathrm{GeV}$," Physics Letters B, vol. 664, pp. 4146, 2008.

[61] J. A. Harvey, C. T. Hill, and R. J. Hill, "Anomaly mediated neutrino-photon interactions at finite baryon density," Physical Review Letters, vol. 99, Article ID 261601, 2007.

[62] S. Gershtein, Y. Y. Komachenko, and M. Y. A. Khlopov, "Production of single photons in the exclusive neutrino process $v N \rightarrow v \gamma N$," Soviet Journal of Nuclear Physics, vol. 33, p. 860, 1981.

[63] R. J. Hill, "Low energy analysis of $v N \rightarrow v N_{\gamma}$ in the standard model," Physical Review D, vol. 81, Article ID 013008, 2010.

[64] R. J. Hill, "Single photon background to $v_{e}$ appearance at MiniBooNE," Physical Review D, vol. 84, Article ID 017501, 2011.

[65] E. Wang, L. Alvarez-Ruso, and J. Nieves, "Photon emission in neutral-current interactions at intermediate energies," Physical Review C, vol. 89, Article ID 015503, 2014.

[66] X. Zhang and B. D. Serot, "Coherent neutrinoproduction of photons and pions in a chiral effective field theory for nuclei," Physical Review C, vol. 86, Article ID 035504, 2012.

[67] X. Zhang and B. D. Serot, "Incoherent neutrinoproduction of photons and pions in a chiral effective field theory for nuclei," Physical Review C, vol. 86, Article ID 035502, 2012.

[68] R. Dharmapalan, I. Stancu, Z. Djurcic et al., "A Proposal for MiniBooNE+: a new investigation of muon neutrino to electron neutrino oscillations with improved sensitivity in an enhanced MiniBooNE experiment," FERMILAB-PROPOSAL-1033, 2013.

[69] X. Zhang and B. D. Serot, "Can neutrino-induced photon production explain the low energy excess in MiniBooNE?" Physics Letters B, vol. 719, pp. 409-414, 2013.

[70] E. Wang, L. Alvarez-Ruso, and J. Nieves, "Single photon events from neutral current interactions at MiniBooNE," http://arxiv .org/abs/1407.6060.

[71] "Neutrino-Nucleus Interactions for Current and Next Generation Neutrino Oscillation Experiments," 2013, http://www.int .washington.edu/PROGRAMS/13-54w.

[72] S. Gninenko, "MiniBooNE anomaly and heavy neutrino decay," Physical Review Letters, vol. 103, Article ID 241802, 2009.

[73] S. N. Gninenko, "Resolution of puzzles from the LSND, KARMEN, and MiniBooNE experiments," Physical Review D, vol. 83, Article ID 015015, 2011.

[74] C. Kullenberg, G. Bassompierre, J. M. Gaillard et al., "A Search for Single Photon Events in Neutrino Interactions," Physics Letters B, vol. 706, pp. 268-275, 2012.

[75] J. Conrad, C. Ignarra, G. Karagiorgi, M. Shaevitz, and J. Spitz, "Sterile neutrino fits to short-baseline neutrino oscillation measurements," Advances in High Energy Physics, vol. 2013, Article ID 163897, 26 pages, 2013. 
[76] P. Ade, N. Aghanim, C. Armitage-Caplan et al., "Planck 2013 results. XVI. Cosmological parameters," 2013, http://arxiv.org/ abs/1303.5076.

[77] G. Mention, M. Fechner, T. Lasserre et al., "The reactor antineutrino anomaly," Physical Review D, vol. 83, Article ID 073006, 2011.

[78] C. Giunti and M. Laveder, "Statistical significance of the gallium anomaly," Physical Review C, vol. 83, Article ID 065504, 2011.

[79] J. Kopp, P. A. N. Machado, M. Maltoni, and T. Schwetz, "Sterile neutrino oscillations: the global picture," Journal of High Energy Physics, vol. 50, 2013.

[80] G. Karagiorgi, "Current and future liquid argon neutrino experiment,” http://arxiv.org/abs/1304.2083.

[81] C. Adams, D. Adams, T. Akiri et al., "The long-baseline neutrino experiment: exploring fundamental symmetries of the universe," 2013, http://arxiv.org/abs/1307.7335.

[82] T. Katori, "MicroBooNE light collection system," Journal of Instrumentation, vol. 8, Article ID C10011, 2013.

[83] B. Baptista, L. Bugel, C. Chiu, J. Conrad, and C. Ignarra, "Benchmarking TPB-coated light guides for liquid argon TPC light detection systems," 2012, http://arxiv.org/abs/1210.3793.

[84] C. Chiu, C. Ignarra, L. Bugel et al., "Environmental efects on TPB wavelength-shifting coatings," http://arxiv.org/abs/1204 .5762 .

[85] B. Jones, J. Van Gemert, J. Conrad, and A. Pla-Dalmau, "Photodegradation mechanisms of tetraphenyl butadiene coatings for liquid argon detectors," Journal of Instrumentation, vol. 8, Article ID P01013, 2013.

[86] T. Briese, L. Bugel, J. Conrad et al., "Testing of cryogenic photomultiplier tubes for the MicroBooNE experiment," Journal of Instrumentation, vol. 8, Article ID T07005, 2013.

[87] A. Curioni, B. Fleming, W. Jaskierny et al., "A regenerable filter for liquid argon purification," Nuclear Instruments and Methods in Physics Research A, vol. 605, pp. 306-311, 2009.

[88] R. Andrews, W. Jaskierny, H. Jostlein, C. Kendziora, and S. Pordes, "A system to test the effects of materials on the electron drift lifetime in liquid argon and observations on the effect of water," Nuclear Instruments and Methods in Physics Research A, vol. 608, pp. 251-258, 2009.

[89] B. Baptista, L. Bugel, C. Chiu et al., "Benchmarking TPB-coated light guides for liquid argon TPC light detection systems," http://arxiv.org/abs/arXiv:1210.3793.

[90] V. A. Kostelecky and S. Samuel, "Spontaneous breaking of Lorentz symmetry in string theory," Physical Review D, vol. 39, article 683, 1989.

[91] V. A. Kostelecky and M. Mewes, "Lorentz and CPT violation in neutrinos," Physical Review D, vol. 69, no. 1, Article ID 016005, 25 pages, 2004.

[92] J. S. Diaz, V. A. Kostelecky, and M. Mewes, "Perturbative Lorentz and CPT violation for neutrino and antineutrino oscillations," Physical Review D, vol. 80, Article ID 076007, 2009.

[93] V. A. Kostelecky and M. Mewes, "Lorentz and CPT violation in the neutrino sector," Physical Review D, vol. 70, Article ID 031902(R), 2004.

[94] J. S. Diaz and V. A. Kostelecky, "Three-parameter Lorentzviolating texture for neutrino mixing," Physics Letters B, vol. 700, no. 1, pp. 25-28, 2011.

[95] J. S. Díaz and A. Kostelecký, "Lorentz- and CPT-violating models for neutrino oscillations," Physical Review D, vol. 85, no. 1, Article ID 016013, 17 pages, 2012.
[96] T. Katori, V. A. Kostelecky, and R. Tayloe, "Global three-parameter model for neutrino oscillations using Lorentz violation," Physical Review D, vol. 74, Article ID 105009, 2006.

[97] L. Auerbach, R. L. Burman, D. O. Caldwell et al., "Tests of Lorentz violation in $\bar{v}_{\mu} \rightarrow \bar{v}_{e}$ oscillations," Physical Review D, vol. 72, Article ID 0506067, 2005.

[98] V. A. Kostelecky and N. Russell, "Data tables for Lorentz and CPT violation," Reviews of Modern Physics, vol. 83, no. 1, pp. 1131, 2011.

[99] D. Colladay and V. A. Kostelecky, "Lorentz-violating extension of the standard model," Physical Review D, vol. 58, Article ID 9809521, 1998.

[100] D. Colladay and V. A. Kostelecky, "CPT violation and the standard model," Physical Review D, vol. 55, pp. 6760-6774, 1997.

[101] V. A. Kostelecký, "Gravity, Lorentz violation, and the standard model," Physical Review D, vol. 69, no. 10, Article ID 105009, 2004.

[102] V. A. Kostelecký and M. Mewes, "Lorentz violation and shortbaseline neutrino experiments," Physical Review D, vol. 70, Article ID 076002, 2004.

[103] A. Aguilar-Arevalo, C. E. Anderson, A. O. Bazarko et al., "Test of Lorentz and CPT violation with short baseline neutrino oscillation excesses," Physics Letters B, vol. 718, no. 4-5, pp. 13031308, 2013.

[104] P. Adamson, C. Andreopoulos, K. E. Arms et al., "Testing Lorentz invariance and CPT conservation with NuMI neutrinos in the MINOS near detector," Physical Review Letters, vol. 101, no. 15, Article ID 151601, 2008.

[105] P. Adamson, D. S. Ayres, G. Barr et al., "Search for Lorentz invariance and CPT violation with muon antineutrinos in the MINOS near detector," Physical Review D, vol. 85, Article ID 031101, 2012.

[106] P. Adamson, D. J. Auty, and D. S. Ayres, "Search for Lorentz invariance and CPT violation with the MINOS far detector," Physical Review Letters, vol. 105, no. 15, Article ID 151601, 2010.

[107] B. Rebel and S. Mufson, "The search for neutrino-antineutrino mixing resulting from Lorentz invariance violation using neutrino interactions in MINOS," Astroparticle Physics, vol. 48, pp. 78-81, 2013.

[108] R. Abbasi, Y. Abdou, and T. Abu-Zayyad, "Search for a Lorentzviolating sidereal signal with atmospheric neutrinos in IceCube," Physical Review D, vol. 82, Article ID 112003, 2010.

[109] Y. Abe, C. Aberle, J. C. dos Anjos et al., "First test of Lorentz violation with a reactor-based antineutrino experiment," Physical Review D, vol. 86, Article ID 112009, 2012.

[110] J. Díaz, T. Katori, J. Spitz, and J. Conrad, "Search for neutrinoantineutrino oscillations with a reactor experiment," Physics Letters B, vol. 727, no. 4-5, pp. 412-416, 2013.

[111] T. Adam, N. Agafonova, A. Aleksandrov et al., "Measurement of the neutrino velocity with the OPERA detector in the CNGS beam," Journal of High Energy Physics, vol. 1210, article 093, 2012.

[112] A. Kostelecký and M. Mewes, "Neutrinos with Lorentzviolating operators of arbitrary dimension," Physical Review D, vol. 85, Article ID 096005, 2012.

[113] B. Batell, M. Pospelov, and A. Ritz, "Exploring portals to a hidden sector through fixed targets," Physical Review D, vol. 80, Article ID 095024, 2009.

[114] P. de Niverville, M. Pospelov, and A. Ritz, "Observing a light dark matter beam with neutrino experiments," Physical Review D, vol. 84, Article ID 075020, 2011. 
[115] P. de Niverville, D. McKeen, and A. Ritz, "Signatures of sub-GeV dark matter beams at neutrino experiments," Physical Review D, vol. 86, Article ID 035022, 2012.

[116] R. Dharmapalan, I. Stancu, R. A. Johnson et al., "A proposal to search for dark matter with MiniBooNE," Fermilab Proposal 1032, 2012.

[117] R. Bernabei, P. Belli, F. Cappella et al., "New results from DAMA/LIBRA," The European Physical Journal C, vol. 67, no. 1-2, pp. 39-49, 2010.

[118] C. Aalseth, P. S. Barbeau, N. S. Bowden et al., "Results from a search for light-mass dark matter with a P-type point contact germaniumdetector," Physical Review Letters, vol. 106, Article ID 131301, 2011.

[119] G. Angloher, M. Bauer, I. Bavykina et al., "Results from 730 $\mathrm{kg}$ days of the CRESST-II dark matter search," The European Physical Journal C, vol. 72, p. 1971, 2012.

[120] R. Agnese, Z. Ahmed, A. J. Anderson et al., "Silicon detector dark matter results from the final exposure of CDMS II," Physical Review Letters, vol. 111, Article ID 251301, 2013.

[121] R. Agnese, A. J. Anderson, M. Asai et al., "Search for lowmass weakly interacting massive particles using voltage-assisted calorimetric ionization detection in the SuperCDMS experiment," Physical Review Letters, vol. 112, no. 4, Article ID 041302, 2014.

[122] G. Bennett, B. Bousquet, H. N. Brown et al., "Final report of the E821 muon anomalous magnetic moment measurement at BNL," Physical Review D, vol. 73, Article ID 072003, 2006.

[123] M. Pospelov, "Secluded U(1) below the weak scale," Physical Review D, vol. 80, Article ID 095002, 2009.

[124] A. Adelmann, J. R. Alonso, W. Barletta et al., "Cost-effective design options for IsoDAR," 2012, http://arxiv.org/abs/1210 .4454 .

[125] D. Adey, S. K. Agarwalla, C. M. Ankenbrandt et al., "nuSTORMneutrinos from STORed muons: proposal to the fermilab PAC," 2013, http://arxiv.org/abs/1308.6822.

[126] Y.-F. Li, J. Cao, Y. Wang, and L. Zhan, "Unambiguous determination of the neutrino mass hierarchy using reactor neutrinos," Physical Review D, vol. 88, Article ID 013008, 2013. 

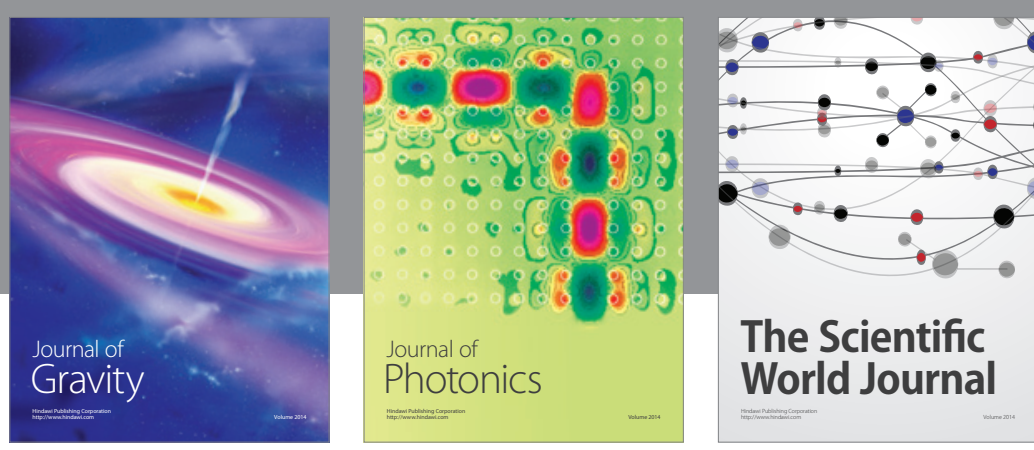

The Scientific World Journal


Submit your manuscripts at

http://www.hindawi.com

nternational Journal of

Statistical Mechanics
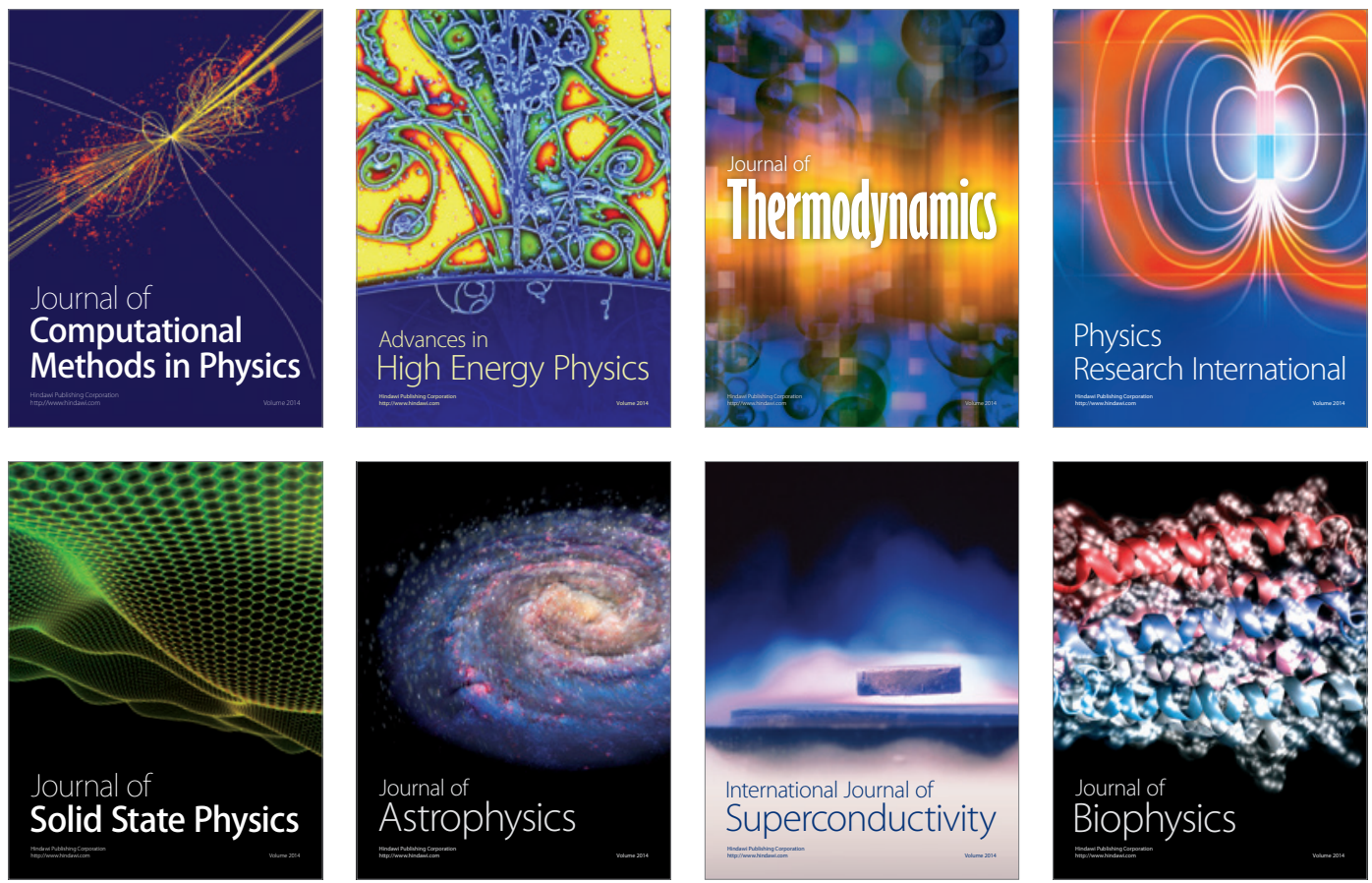
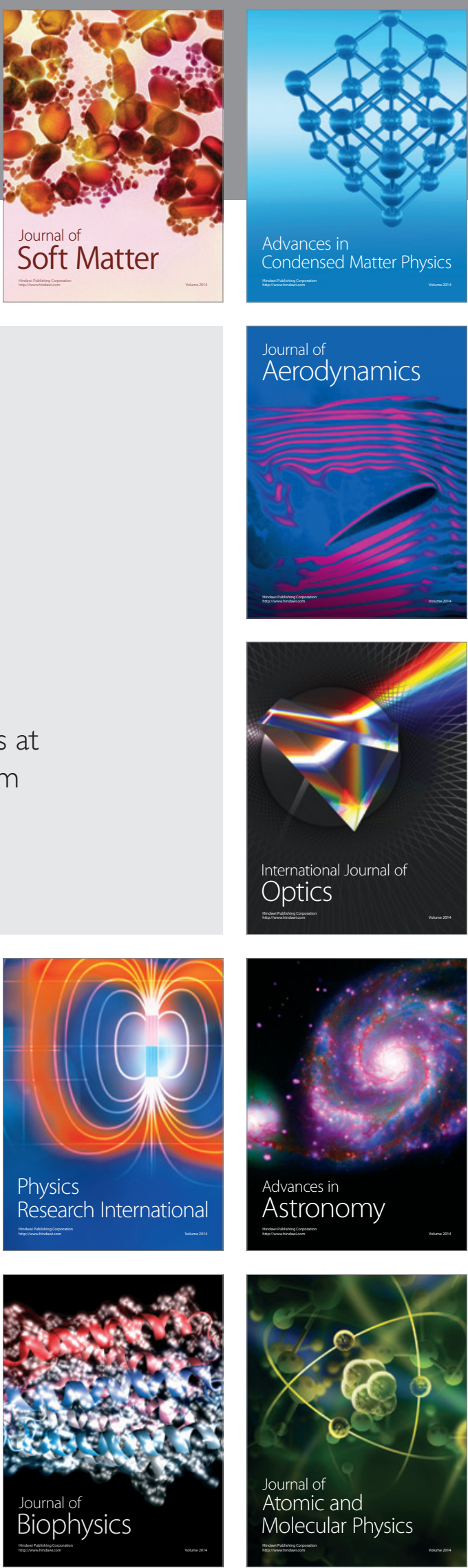